\title{
Theory of resonance influence of sawtooth crashes on ions with large orbit width
}

\author{
Ya. I. Kolesnichenko*, V. V. Lutsenko*, R. B. White ${ }^{* *}$, and Yu. V. Yakovenko* \\ *Scientific Centre "Institute for Nuclear Research", Kyiv, 252650, Ukraine \\ ${ }^{* *}$ Princeton Plasma Physics Laboratory, P.O.Box 451, Princeton, NJ, 08543, USA
}

(January 20, 1998)

\begin{abstract}
The role of resonances in the sawtooth-crash-induced redistribution of fast ions is investigated. In particular, the conditions of wave-particle resonant interaction in the presence of the equilibrium electric field and the mode rotation are obtained, and effects of sawteeth on the resonant particles with arbitrary width of non-perturbed orbits are studied. It is found that resonances play the dominant role in the transport of ions having sufficiently high energy. It is shown that the resonance regions may overlap, in which case the resonant particles may constitute the main fraction of the fast ion population in the sawtooth mixing region. The behavior of the resonant particles is studied both by constructing a Poincaré map and analytically, by means of the adiabatic invariant derived in this paper and calculation of the characteristic frequencies of the particle motion.
\end{abstract}




\section{INTRODUCTION}

Two years ago an approach to the description of fast ion transport induced by crashes of sawtooth oscillations has been suggested, which revealed important features of such transport. ${ }^{1}$ It was found that there is a critical energy, $\mathcal{E}_{\text {crit }}$, such that when $\mathcal{E}>\mathcal{E}_{\text {crit }}$ the radial redistribution of ions depends essentially on their pitch-angles. In particular, when $\mathcal{E}>\mathcal{E}_{\text {crit }}$, the bulk of trapped ions with small banana width are weakly sensitive to a crash, whereas circulating particles are strongly redistributed.

These theoretical predictions are in agreement with experimental observations ${ }^{2-6}$ on the Tokamak Fusion Test Reactor (TFTR) ${ }^{7}$ and the Joint European Torus (JET). ${ }^{8}$

The critical energy is determined from the condition that the crash duration is equal to the period of the toroidal precession of the well-trapped fast ions, and can be written as follows:

$$
\mathcal{E}_{\text {crit }}=2 \pi M r_{s} R \omega_{B} / \tau_{c r},
$$

where $\tau_{c r}$ is the crash duration, $r_{s}$ is the radius of the $q=1$ surface, $M$ is the ion mass, and $\omega_{B}$ is the cyclotron frequency. The $\mathcal{E}_{\text {crit }}$ exists due to the fact that the toroidal precession tends to decorrelate the phase of interaction of particles and the electromagnetic helical perturbation associated with the crash.

It is clear that the precession is negligible for $\mathcal{E} \ll \mathcal{E}_{\text {crit }}$. Therefore, slow particles (both circulating and trapped) are strongly redistributed by the crash, being 'attached' to the evolving flux-surfaces. Their redistribution can be described by earlier theory 9 . The motion of the 'flux-surface-attached' particles can be consid-

ered as a result of the $\vec{E} \times \vec{B}$ drift, where $\vec{E}$ is the electric field generated due to evolution of the magnetic configuration during the crash, and $\vec{B}$ is the magnetic field. ${ }^{1,10}$ 
On the other hand, when $\mathcal{E} \gg \mathcal{E}_{\text {crit }}$, an essential fraction of the fast ion population may have non-standard orbits, $\Delta r_{b} \gtrsim r$, where $\Delta r_{b}$ is the orbit width in the absence of sawteeth, $r$ is the radial coordinate. In particular, most of fusionproduced alpha particles in the sawtooth mixing region of TFTR and JET are characterized by non-standard orbits. One can expect that certain groups of these particles will be sensitive to the sawtooth crash in spite of the strong precession. Indeed, when $\Delta r_{b} \gtrsim r$, the bounce period is of the order of the period of the toroidal precession. Therefore, resonance between the poloidal and toroidal motion is possible. The corresponding resonance condition can be written as follows:

$$
s \omega_{b}=n \omega_{\phi},
$$

where $\omega_{b}$ is the bounce/transit frequency, $\omega_{\phi}$ is the frequency of the particle motion in the toroidal direction, $n$ is the toroidal mode number, and $s$ is an integer. For the particles satisfying Eq. (2), the toroidal precession will not decorrelate the phase of the wave-particle interaction, at least when the perturbation frequency is negligible, therefore their behavior will differ from that of other fast ions.

Note that the resonance condition (2) can be satisfied also when $\Delta r_{b} \ll r$ but only in the degenerate case of $s=0$. In this case, Eq. (2) singles out a narrow group of marginally trapped ions, this group being the same for any mode number and consisting of the particles with the trapping parameter $\kappa \approx 0.9$, where $\kappa \equiv\left\{\left[\mathcal{E} R_{0}+\mu B_{0}\left(r-R_{0}\right)\right] /\left(2 \mu B_{0} r\right)\right\}^{1 / 2}, \mu$ is the particle magnetic moment, $B_{0} \equiv$ $B(r=0), R_{0} \equiv R(r=0)$ is the radius of the unperturbed magnetic axis, and $R$ is the distance to the axis of symmetry. The behavior of these particles was studied in a recent work, ${ }^{11}$ where it was shown that they move along superbanana orbits and, therefore, their orbit width essentially exceeds that of other particles.

The main purpose of the present work is to investigate effects of resonances on the sawtooth induced transport of fast ions with $\mathcal{E}>\mathcal{E}_{\text {crit }}, \Delta r_{b} \gtrsim r$. In addition, this work is aimed to elucidate the role of finite wave frequency and the associated mode rotation, which were neglected in previous studies. 
The work is organized as follows. In Sec. II, the harmonic spectrum of the electromagnetic perturbation associated with a sawtooth crash is analyzed. Sec. III deals with a linear theory of the resonant wave-particle interaction, i.e., a theory where the change of the characteristic frequencies of particle motion is neglected. Non-linear behavior of fast ions near the resonance drift surfaces is studied in Sec. IV. The summary of the obtained results and the drawn conclusions are given in Sec. V. Features of motion of particles with arbitrary orbit width in the absence of sawtooth oscillations are analyzed in Appendix A. Appendix B contains the derivation of the adiabatic invariant used for non-linear analysis of the resonances.

\section{ELECTROMAGNETIC FIELD DURING A CRASH}

Experiments indicate that a sawtooth crash is associated with development of the $m=n=1$ helical perturbation. In spite of this, higher harmonics with $m=n$ are to be present, too. The reason is that the perturbation having initially the sinusoidal form becomes non-harmonic on the non-linear stage of the instability.

In order to demonstrate a contribution of harmonics with $m=n>1$, we expand a perturbed quantity $X$ in the Fourier series:

$$
X(r, \alpha, t)=\sum_{n} X_{n}(r, t) \exp (i n \alpha-i \omega t), \quad m=n
$$

where the helical coordinate system is used, namely, $\left(x^{1}, x^{2}, x^{3}\right)=(r, \alpha \equiv \vartheta-\varphi, \varphi)$; $\vartheta$ and $\varphi$ are the poloidal and toroidal coordinates, respectively; $\omega$ is the wave frequency. Then we assume that the toroidal magnetic field is not perturbed by the crash and take into account that the perturbed electromagnetic field is described in terms of the helical magnetic flux, $\Psi$, as follows: ${ }^{9}$

$$
\vec{B}=B^{3} \vec{e}_{3}+\vec{e}^{3} \times \nabla \Psi, \quad \vec{E}=\frac{1}{c} \frac{\partial \Psi}{\partial t} \vec{e}^{3}-\nabla \Phi
$$

Here the base co- and contravariant vectors $\vec{e}_{j}$ and $\vec{e}^{j}(j=1,2,3)$ are used, $B^{3}=$ $B_{0} R_{0} / R^{2}$, and $\Phi(r, \alpha, t)$ is the electric potential related to $\Psi$ by the equation $\vec{E} \cdot \vec{B}=$ 0 , which can be written in the form: ${ }^{1}$ 


$$
\frac{\partial \Psi}{\partial t}+\frac{c R}{B \sqrt{g}}\left(\frac{\partial \Phi}{\partial r} \frac{\partial \Psi}{\partial \alpha}-\frac{\partial \Phi}{\partial \alpha} \frac{\partial \Psi}{\partial r}\right)=0
$$

where $g$ is the determinant of the metric tensor $g_{i j}$. The temporal evolution of $\Psi$ determines the kind of a crash.

Let us take the analytical expression for $\Psi(r, \alpha, t)$ suggested in Ref. ${ }^{9}$ for modeling the Kadomtsev-type crash. Then, after calculations, we find that the harmonics with $m=n=2$ and 3 essentially contribute to the electromagnetic field during a considerable part of the crash. In particular, at the final stage of the reconnection of the magnetic field lines, the calculated amplitudes of these harmonics are $\Phi_{2} \sim 2 \Phi_{3} \sim \Phi_{1} / 3$ and $\Psi_{2} \sim 2 \Psi_{3} \sim \Psi_{1} / 2$, see Fig. 1. Probably, the role of the harmonics $m=n \neq 1$ is even more essential because this model represents the simplest pattern of the plasma flow during a crash. Moreover, in reality a crash is accompanied also by $m \neq n$ harmonics, first of all, $m=2, n=1$. These harmonics contribute to the transport of fast circulating ions, leading to stochasticity

of the magnetic field lines. ${ }^{12}$ However, the harmonics with $n=1$ cannot satisfy the resonance condition (2) (see also Sec. III) because typically $\omega_{b} / \omega_{\phi} \geq 1$. In this work we ignore the effects of stochasticity of the magnetic field lines, which gives us grounds to assume that $m=n$.

\section{WAVE-PARTICLE RESONANCE IN THE PRESENCE OF EQUILIBRIUM ELECTRIC FIELD AND MODE ROTATION}

It was shown in Ref. ${ }^{11}$ that the ions of narrow banana width, $\Delta r_{b} \ll r$, and the trapping parameter $\kappa \approx 0.9$ are sensitive to the crash even when $\mathcal{E} \gg \mathcal{E}_{\text {crit }}$. Difference in behavior of these and other particles with small $\Delta r_{b}$ is associated with the fact that the particles with $\kappa=0.91$ are characterized by very small toroidal precession frequency, due to which they are 'resonant', being trapped or marginally untrapped with respect to the helical perturbation of the electromagnetic field with $\omega=0$. The particles trapped in the perturbation move along superbanana 
orbits, oscillating in the radial and toroidal directions near the resonance radius $r_{r}$ determined by the equation $\kappa\left(r_{r}\right)=0.91$.

But, in general, the condition of small banana width, $\Delta r_{b} \ll r$, is difficult to reconcile with the condition $\mathcal{E} \gg \mathcal{E}_{\text {crit }}$. Therefore, it is of interest to find high energy resonant particles with arbitrary orbit width and investigate their behavior. In this section we make the first step in this direction, assuming that frequencies of the particle motion weakly change during the crash, which implies that the effect of the crash on the particles is small. This assumption corresponds to the approximation of linear resonance in the theory of non-linear oscillations. ${ }^{13}$ In this sense, our analysis is 'linear' in spite of the fact that we make no restrictions on amplitudes of perturbations of the electromagnetic field. Linear analysis enables us to obtain the resonance condition and demonstrate that resonant and non-resonant particles are affected by the crash in different ways. Moreover, it enables us to make conclusions concerning the role of the mode rotation and the equilibrium radial electric field the factors which always take place but were ignored in previous studies.

We proceed from the following equations describing the particle behavior during the crash:

$$
\begin{aligned}
& \dot{\vec{r}}=\vec{v}_{\|}+\vec{v}_{D}+\vec{v}_{E}, \\
& \dot{\mathcal{E}}=\vec{E} \cdot\left(\vec{v}_{\|}+\vec{v}_{D}\right),
\end{aligned}
$$

where the dot denotes the time derivative along the guiding center orbit, $\vec{v}_{\|} \equiv$ $v_{\|} \vec{B} / B, v_{\|}=\left\{(2 / M)\left[\mathcal{E}-\mu B_{0}(1-\epsilon \cos \vartheta)\right]\right\}^{1 / 2}, \vec{v}_{D} \equiv-\vec{v}_{\|} \times \nabla\left(v_{\|} / \omega_{B}\right), \vec{v}_{E} \equiv$ $c \vec{E} \times \vec{B} /|B|^{2}, \epsilon=r / R_{0}$.

We assume that the crash duration exceeds the particle bounce/transit time period $\left(\tau_{b}\right)$. Then the particle displacement can be characterized by the change of the canonical angular momentum, which motives us to proceed from Eqs. (6), (7) to the following equations:

$$
\dot{J}_{T}=\frac{e v \|}{c R} \frac{\partial \Psi}{\partial \alpha}+e \frac{\partial \Phi}{\partial \alpha}
$$




$$
\dot{\alpha}=v_{\|}^{2}+v_{D}^{2}+v_{E}^{2}=\frac{v_{\|}}{R}\left(\frac{B_{\vartheta}}{\epsilon B}-1\right)+\frac{v_{D \vartheta}}{r}+\frac{v_{E \vartheta}}{r},
$$

where the superscript ' 2 ' labels the corresponding contravariant components, $J_{T}$ is the canonical angular momentum given by

$$
J_{T}=-\frac{e}{c} \Psi_{p}+M v_{\|} R
$$

$\Psi_{p}=\Psi+F(r)$ is the poloidal magnetic flux, and $F(r) \equiv \int_{0}^{r} d r \sqrt{g} B^{3}$ is the toroidal magnetic flux.

The equation ( 8 ) is linear in the perturbation amplitude. Therefore, its formal solution can be written as follows:

$$
\Delta J_{T}=\sum_{n} \int_{0}^{\tau_{c r}} d t^{\prime} A_{n}\left(t^{\prime}\right) \exp \left[i \int_{0}^{t^{\prime}} d t^{\prime \prime}(n \dot{\alpha}-\omega)\right]
$$

where $A_{n}$ is a quantity proportional to the perturbation amplitude.

$\dot{\alpha}$ entering Eq. (11) is determined by the right hand side of Eq. (9), which can be simplified. Indeed, using Eq. (5), we find that

$$
\Phi(r, \Psi, t)=\int_{r_{*}}^{r} d r \frac{B^{3}}{c B^{1}} \frac{\partial \Psi}{\partial t}+\Phi_{*}
$$

where $\Phi_{*}=\Phi\left(r_{*}, \Psi, t\right), r_{*}$ is a point on the flux surface $\Psi$. On the other hand, due to Eq. (3),

$$
\frac{\partial \Psi}{\partial t}=\left.\frac{\partial \Psi}{\partial t}\right|_{n \alpha-\omega t}-\frac{\omega}{n} \frac{\partial \Psi}{\partial \alpha}
$$

Taking into account that $B^{1}=-(1 / \sqrt{g}) \partial \Psi / \partial \alpha$, we present $\Phi$ as follows:

$$
\Phi(r, \Psi, t)=\hat{\Phi}+\Phi_{1}+\Phi_{\omega}
$$

where

$$
\begin{gathered}
\hat{\Phi}=\left.\int_{r_{1}}^{r} d r \frac{B^{3}}{c B^{1}} \frac{\partial \Psi}{\partial t}\right|_{n \alpha-\omega t}, \\
\Phi_{\omega}=\frac{\omega}{n c} \int_{r_{1}}^{r} d r \sqrt{g} B^{3},
\end{gathered}
$$


$\Phi_{\omega}$ is the part of the potential associated with the mode rotation. Correspondingly, $v_{E}^{2}=\hat{v}_{E}^{2}+v_{E *}^{2}+v_{E \omega}^{2}$, where $v_{E \omega}^{2}=\omega / n$. Therefore,

$$
n \dot{\alpha}-\omega=n\left(v_{\|}^{2}+v_{D}^{2}+\hat{v}_{E}^{2}+v_{E *}^{2}\right)
$$

The quantity $\vec{v}_{E *}$ describes the plasma rotation along the perturbed flux surfaces. One can expect that both before and during the crash $\Phi_{*} \sim T / e$, where $T$ is the plasma temperature. This is because the crash is presumably associated with development of the tearing instability accompanied by large-scale plasma motion (note that, in general, $\Phi_{*}$ differs from $T / e$; for instance, during fishbone oscillations may essentially exceed the thermal level to compensate for the large $\left.\Phi_{\omega}\right)$. Assuming that this is the case and taking $\mathcal{E} \gg T \epsilon^{-1}$, we neglect $v_{E *}^{2}$, which is small compared to $v_{D}^{2}$. We assume also that $\mathcal{E} \gg \mathcal{E}_{\text {crit }}$, when $\hat{v}_{E}^{2}$ is also small compared to $v_{D}^{2}$. Under these assumptions, $n \dot{\alpha}-\omega=n\left(v_{\|}^{2}+v_{D}^{2}\right)$. The contribution of the perturbation to $\vec{v}_{D}$ is negligible. The perturbed part of $v_{\|}^{2}$ is also negligible for all particles except the narrow-orbit circulating particles (for the latter, the perturbed part of $v_{\|}^{2}$ is small only immediately after the beginning of the crash). Therefore, below the contribution of the perturbation to $n\left(v_{\|}^{2}+v_{D}^{2}\right)$ is neglected.

Neglecting the perturbation, we assume that $n\left(v_{\|}^{2}+v_{D}^{2}\right)$ is a periodic function of time on the particle orbit. In this case, Eq. (11) can be written as:

$$
\Delta J_{T}=\sum_{n} \sum_{p=0}^{N} \exp \left(i p \Omega \tau_{b}\right) \int_{0}^{\tau_{b}} d \tau A_{n}\left(\tau+p \tau_{b}\right) \exp \left[i \int_{0}^{\tau} d t^{\prime}\left(n\left(v_{\|}^{2}+v_{D}^{2}\right)\right]+\delta J_{T},\right.
$$

where

$$
\Omega=n\left\langle v_{\|}^{2}+v_{D}^{2}\right\rangle
$$

the brackets $\langle\ldots\rangle$ mean bounce averaging, $N$ is the integer part of the ratio $\tau_{c r} / \tau_{b}$, $\delta J_{T}$ is a banana displacement for $\Delta t<\tau_{b}$.

It follows from Eq. (18) that $\Delta J_{T}$ is maximum when either

$$
\Omega \tau_{c r} \ll 2 \pi
$$




$$
\Omega \tau_{b}=2 \pi s
$$

The equations $(19)$ and $(20)$ yield $\mathcal{E}_{\text {crit }}$ for trapped particles with narrow orbits. We conclude from this that, when $\mathcal{E}<\mathcal{E}_{\text {crit }}$, the coherent interaction between narrow-orbit trapped particles and the waves takes place, which provides the particle redistribution. The equation (20) cannot be satisfied for circulating particles with narrow orbits. However, our analysis is not valid for them. Such particles are strongly redistributed by the crash because they are frozen in the magnetic field and follow the evolving flux surfaces even when $\mathcal{E}>\mathcal{E}_{\text {crit }}{ }^{1}$

The equation (21) represents the condition of wave-particle resonance, which is well-known in the theory of plasma instabilities. In general, it can be written in the form:

$$
\omega+n \omega_{\phi}+(C n-s) \omega_{b}=0,
$$

where $C$ equals unity for circulating particles and zero for trapped ones. This equation determines the resonance curves $\mathcal{E}(\mu)$ at a given point $r, \vartheta$ or the resonance value of $J_{T}$ and, thus, the resonance drift surface $r(\vartheta)$ for a particle with given $\mathcal{E}, \mu$. When $\omega \ll \omega_{b}$, it, in fact, coincides with Eq. (2) (the only difference is that $s$ has been redefined for circulating particles). Due to Eq. (19), the resonance condition can also be written for all particles except for the narrow-orbit circulating ones as follows:

$$
n\left\langle\frac{v_{D \vartheta}}{r}\right\rangle \approx s w_{b}
$$

This equation, as well as Eqs. (20) and (21) with $\Omega$ given by Eq. (19), depend on $\omega$ and $E_{\omega r}$ only through the shape of the orbits. Therefore, we can conclude that the resonance condition for the trapped particles with $\Delta r_{b} \ll r$ and, which is more important, the magnitude of $\mathcal{E}_{\text {crit }}$, do not depend on the mode rotation and the equilibrium electric field.

The derived feature is a consequence of the assumption that the helical symmetry is conserved during the crash, which implies that fast ions do not affect the 
plasma flow. However, difference in the redistribution of different groups of particles during the crash (trapped and circulating particles, resonant and non-resonant particles etc.) may lead to violation of the helical symmetry. It is clear that this effect may be essential when the number of fast ions is not very small. Moreover, it is known that the presence of fast ions with a dominant trapped-ion population can even stabilize sawtooth oscillations ${ }^{14}$ provided that their pressure is sufficiently high (the corresponding condition can be found in, e.g., Ref. ${ }^{15}$ ).

It follows from Eq. (18) that the effect of resonances is the strongest when $\tau_{c r} \gg \tau_{b}$. On the contrary, when $\tau_{c r} \ll \tau_{b}$ then there is no difference between the resonant and non-resonant particles.

Note that the rate of the energy change of the resonant particles is much less than that of the other particles. This can be shown as follows. Write Eq. (7) in the form

$$
\dot{\mathcal{E}}=e \vec{v} \cdot \vec{E}=-e \frac{d \Phi}{d t}+e \frac{\partial \Phi}{\partial t}+e v^{3} E_{3}
$$

This equation yields

$$
\int_{t_{1}}^{t_{2}} d t \dot{\mathcal{E}}=-e\left(\Phi_{2}-\Phi_{1}\right)+O\left(\tau_{b} / \tau_{c r}\right)
$$

where $t_{2}=t_{1}+\tau_{b}$, the subscripts ' 1 ' and ' 2 ' label the points on a particle orbit at $t=t_{1}$ and $t=t_{2}$, respectively. Using Eqs. (21) and $\Omega \simeq\langle\dot{\alpha}\rangle-\omega$, we find:

$$
n\left(\alpha_{2}-\alpha_{1}\right)-\omega \tau_{b}=2 \pi s .
$$

It follows from Eqs. $(25),(26)$ that $\Phi_{2}-\Phi_{1} \approx 0$ and, thus, $\langle\dot{\mathcal{E}}\rangle \approx 0$ for the resonant particles.

According to Eq. (18), the displacement of the resonant particles tends to infinity when $\tau_{c r} \rightarrow \infty$. The reason of this is that we neglected violation of the resonance during the particle motion. In reality, however, the displacement of resonant particles depends on the wave-particle interaction not only on the resonance surface but also beyond it and can be found from a non-linear theory. 


\section{NON-LINEAR BEHAVIOR OF RESONANT PARTICLES}

In Sec. III we have found the conditions of resonant interaction between particles and a perturbed electromagnetic field. To understand whether the resonances are important, one should answer two questions. First, how many particles are resonant? Second, how do the resonances affect the particles? The answers depend on consistency of the frequencies of particle motion with the resonance condition (22), results of influence of a crash on resonant particles, and the effective width of the resonances.

Below we consider these questions, taking into account that typically $|q-1| \ll 1$ in the sawtooth mixing region. This gives us grounds to consider the magnetic field with $q(r) \equiv 1$ as the unperturbed field and the contribution of $\Psi$ as a perturbation. ${ }^{1}$ In addition, we assume that $\omega=0$ and $E_{r 0}=0$. This assumption is justified because experiments indicate that typical frequencies of the mode rotation during sawtooth crashes are small compared to the bounce frequency of fast ions. On the other hand, our analysis in Sec. III shows that the wave frequency is associated with the equilibrium electric field. Thus, if $\omega$ is negligible, the equilibrium electric field can be neglected, too.

\section{A. Resonant particles}

We begin with a consideration of features of the unperturbed motion of the particles, such as the orbit shape and the frequencies of the motion, $\omega_{b}$ and $\omega_{\phi}$.

Topological classification of particles with arbitrary orbit width was given in,

e.g., Refs. ${ }^{16-20}$. In addition, an analytical expression for $\omega_{b}$ of such particles has been obtained in Ref. ${ }^{19}$. The general conclusion which follows from these works is that the particle orbits and the characteristic frequencies of their motion depend on all three constants of motion of the particle, $\mathcal{E}, \mu$, and $J_{T}$, as well as on parameters of the magnetic configuration, such as the $q(r)$ profile, the Shafranov shift, and the flux surface shape. However, the problem is considerably simplified if we are interested 
in behavior of particles in the central part of a plasma and, therefore, can assume that the flux surfaces are concentric ellipses of constant ellipticity $(k)$ and $q(r) \approx$ const. In this case, both the orbit topology ${ }^{18}$ and the ratio $\omega_{\phi} / \omega_{b}^{21}$ are completely determined by two dimensionless constants of motion, $L \equiv\left(1-\mu B_{0} / \mathcal{E}\right) \nu^{-1}$ and $P \equiv-2 c J_{T} q /\left(k e B_{0} R_{0}^{2} \nu^{2}\right)$, where $\nu \equiv\left[2 q \rho /\left(k R_{0}\right)\right]^{2 / 3}, \rho$ is the Larmor radius at $R=R_{0}$. The corresponding analysis is given in Appendix A. Note that the analysis is more general than it is required for our purposes as it does not use the assumption $q=1$. The resulting orbit classification is presented in Fig. 2, and level contours of the calculated ratio of the frequencies of the toroidal and poloidal motion, in Fig. 3.

It follows from Fig. 3 that there are particles with the values $\omega_{\phi} / \omega_{b}=$ $0,1 / 3,1 / 2,2 / 3,1$, which are resonant to the dominant modes of the sawtooth crash, $m=n=1,2,3$. The orbits of the resonant particles are characterized by large width $\Delta r_{b} \sim r$, and have a 'potato'-like shape. The exception is the $\omega_{\phi}=0$ resonance, which is possible also for $\Delta r_{b} \ll r$, when it is relevant to marginally trapped particles with the trapping parameter $\kappa=0.91$. When the ion energy is sufficiently high, $\Delta r_{b} \sim r_{s}$, resonant particles with large orbit width occupy a major part of the sawtooth mixing region.

To study the particle motion near the resonances under influence of a perturbation, we use two approaches. First, we use a guiding center code to construct a Poincaré map of the particle motion near a resonance. Second, we apply an analytical approach.

\section{B. Description of resonant particles by the Poincaré map}

To construct Poincaré maps, we integrate Eqs. (6), (7) and plot the points where particle trajectories intersect the equatorial plane of the torus.

We assume that either a magnetic or an electrostatic perturbation is present, i.e., either $\Psi \neq 0, \Phi=0$ or $\Phi \neq 0, \Psi=0$. This assumption simplifies the 
analysis and, at the same time, enables to understand the role of the longitudinal motion and the $\vec{E} \times \vec{B}$ drift. We take the perturbations in the following form, which describes superposition of the $m=n=2$ and $m=n=3$ modes:

$$
X=X_{2 \max } f_{2}(r) \exp (2 i \alpha)+X_{3 \max } f_{3}(r) \exp (3 i \alpha)
$$

Here $X$ is either $\Psi$ or $\Phi ; X_{2 \max }$ and $X_{3 \max }$ are the amplitudes of the corresponding harmonics at the points where they are maximum; the functions $f_{2}(r)=4\left(r / r_{m i x}\right)^{2}\left[1-\left(r / r_{m i x}\right)^{2}\right]$ and $f_{3}(r)=(25 / 6)(5 / 3)^{1 / 2}\left(r / r_{m i x}\right)^{3}\left[1-\left(r / r_{m i x}\right)^{2}\right]$ model the radial profiles of these harmonics, $\max f_{2}(r)=\max f_{3}(r)=1$.

The Poincaré maps obtained for various perturbation amplitudes are shown in Fig. 4. Fig. 4 (a) demonstrates creation of a superbanana orbit (Chirikov island) due to the resonance $1 / 3$ in the presence of an electrostatic perturbation. The orbit is formed as a result of simultaneous action of the radial $\vec{E} \times \vec{B}$ drift caused by the perturbation and the toroidal precession. Adding the $m=n=2$ perturbation of a small amplitude affects the particle behavior near the resonance $1 / 3$, leading to appearance of secondary islands and a stochastic layer near the island separatrix, see Fig. 4 (b). A similar stochastic layer arises also in the case of a single harmonic with larger amplitude, see Fig. 5. An example of the superbananas resulting from a magnetic perturbation is shown in Fig. $4(\mathrm{c})$. Note that the effect of the magnetic perturbations was stronger than that of electrostatic perturbations in the analyzed cases of the mode amplitudes that are expected for the Kadomtsev crash (see Sec. II).

All particles that intersect the resonant drift surface are trapped with respect to the perturbation and move along superbanana orbits. Depending on the wave phase at the points of the intersection, they are characterized by different extent of trapping in the wave and, correspondingly, different superbanana width. The figure 5, where the Poincaré map is shown for several particles characterized by various wave phases at the intersection with the resonance surface, demonstrates this. In addition, Fig. 5 shows orbits of the particles that are not trapped in the 
wave. We observe that the orbit width of most of superbananas, $\Delta r_{s b}$, is much greater than the width of the passing particles. However, the superbanana width is less than the width of unperturbed orbits of the considered particles.

In spite of the fact that $\Delta r_{s b} \ll r_{m i x}$, motion along the superbanana orbits must essentially redistribute the considered particles during the crash because $\Delta r_{b} \sim$ $r_{m i x}$. Therefore, we can conclude that the resonance interaction is a dominant mechanism of the crash-induced redistribution of fast ions with $\Delta r_{b} \sim r_{m i x}$. This conclusion is true provided the period of the superbanana motion, $\tau_{s b}$, is less or, at least, of the order of $\tau_{c r}$, which was the case in our calculations. As a result of redistribution of resonant particles, plateaus on the radial distribution of bananas near the resonance values of $J_{T}$ must be formed. Note that this pattern differs from redistribution of particles with $\mathcal{E} \ll \mathcal{E}_{c r i t}, \Delta r_{b} \ll r_{s}$, in which case the crashinduced displacement of bananas essentially exceeds $\Delta r_{b}$.

Stochasticity near the island separatrix is a factor that enhances transport of resonant particles. However, the presented Poincaré maps show the particle motion in the electromagnetic field of steady-state perturbations for thousands of bounce periods. Therefore, one can expect that, in reality, finite crash duration will prevent the stochastic motion.

\section{Analytical description of motion of resonant particles}

Due to Poincaré maps, we investigated general properties of particle motion near a resonance. However, generating a Poincaré map is rather time-consuming and, therefore, not convenient for studying the dependence of the resonance width on

the parameters $P$ and $L$. For this reason, below we present an analytical approach based on the adiabatic invariant derived in Appendix B.

We consider particle motion near the resonance surface $\omega_{\phi} / \omega_{b}=s / n$, neglecting the influence of harmonics with the toroidal mode number not equal to $n$ and assuming that perturbations weakly change constants of the unperturbed motion 
for one bounce period.

Let us introduce new coordinates associated with the guiding-center motion. The unperturbed particle motion is characterized by three constants of motion $(\mathrm{COM}): \mathcal{E}, \mu$, and $J$, where $J=\left.J_{T}\right|_{\Psi=0}=-(e / c) F+M v_{\|} R$. Given $\mathcal{E}$ and $\mu, J$ can be considered as a function of $r$ and $\vartheta$. As a result, an unperturbed trajectory lies on a torus (drift surface) determined by the equation $J(r, \vartheta)=$ const. It is convenient to replace the poloidal and toroidal coordinates $\vartheta$ and $\varphi$ by the canonical angular coordinates associated with the torus, $\theta(\vartheta, J, \mu, \mathcal{E})$ and $\phi(\varphi, \vartheta, J, \mu, \mathcal{E})$, defined as follows:

$$
\theta=\omega_{b} \int_{0}^{\vartheta} d \vartheta / \dot{\vartheta}, \quad \phi=\varphi+\chi(\vartheta)
$$

where $\chi \equiv \int_{0}^{\vartheta} d \vartheta\left(\omega_{\phi}-\dot{\varphi}\right) / \dot{\vartheta}$ is a continuous periodic function of $\vartheta$, the integrals are taken along the orbit $J(r, \vartheta)=$ const, $\dot{\vartheta}(\vartheta, J, \mathcal{E}, \mu)=\left(\vec{v}_{\|}+\vec{v}_{D}\right) \cdot \nabla \vartheta, \dot{\varphi}(\vartheta, J, \mathcal{E}, \mu)=$ $\left(\vec{v}_{\|}+\vec{v}_{D}\right) \cdot \nabla \varphi \approx v_{\|} / R$, and the poloidal and toroidal frequencies are given by

$$
\begin{gathered}
\omega_{b}=2 \pi(\oint d t)^{-1}=2 \pi\left[\oint d \vartheta(\dot{\vartheta})^{-1}\right]^{-1}, \\
\omega_{\phi}=\frac{\omega_{b}}{2 \pi} \oint d t \dot{\varphi}=\frac{\omega_{b}}{2 \pi} \oint \frac{d \vartheta}{\dot{\vartheta}} \frac{v_{\|}}{R} .
\end{gathered}
$$

The coordinates $\theta$ and $\phi$ were introduced for analyzing resonant interaction of particles with waves. ${ }^{22}$ Their convenience is associated with the fact that $\dot{\theta}$ and $\dot{\phi}$ in the absence of a perturbation are constants on a drift surface, $\dot{\theta}=\omega_{b}(J, \mathcal{E}, \mu)$, $\dot{\phi}=\omega_{\phi}(J, \mathcal{E}, \mu)$.

One can see that, in addition to $J, \mathcal{E}$ and $\mu$, there is one more constant of the unperturbed motion, $\hat{\phi} \equiv n \phi-s \theta$, at the resonant surface. Then, in the presence of a perturbation, the following quantity, which will be called the "resonance invariant', is an adiabatic invariant of the particle motion near the resonance (see Appendix B):

$$
I(\hat{\phi}, J, \mathcal{E}, \mu)=\bar{I}(J, \mathcal{E}, \mu)+\tilde{I}(\hat{\phi}, J, \mathcal{E}, \mu)
$$


Here

$$
\bar{I}=J_{p}+\frac{s}{n} J
$$

is the part of $I$ independent of perturbations; $J_{p}$ is the poloidal action ${ }^{22}$ given by

$$
\begin{gathered}
J_{p}=\frac{1}{2 \pi} \oint d \vartheta \frac{e}{c} F(r) ; \\
\tilde{I}=\frac{1}{2 \pi} \oint \frac{d \vartheta}{\dot{\vartheta}}\left(-\frac{e v \|}{c R} \Psi-e \Phi\right) ;
\end{gathered}
$$

and the integral in Eq. (34) is taken at constant $\hat{\phi}, J, \mathcal{E}$ and $\mu$. One can show (the proof will be presented elsewhere) that the equations of the bounce-averaged motion near the resonance can be written in terms of the resonance invariant as follows:

$$
\dot{\hat{\phi}}=-n \omega_{b} \frac{\partial I}{\partial J}, \quad \dot{J}=n \omega_{b} \frac{\partial I}{\partial \hat{\phi}} .
$$

We find the particle orbits, using the following procedure. We take the potentials as

$$
\Psi=\Psi_{n}(r, \vartheta) \exp (i n \varphi), \quad \Phi=\Phi_{n}(r, \vartheta) \exp (i n \varphi)
$$

(note that here we do not have to restrict ourselves to the modes with $m=n$ ). Substituting Eqs. (36) to Eq. (34) and proceeding to the variables $\hat{\phi}$ and $\theta$, we present the oscillating part of the resonance invariant $I$ as:

$$
\tilde{I}=\Delta I \exp (i \hat{\phi})
$$

where

$$
\Delta I=\frac{1}{2 \pi} \oint \frac{d \vartheta}{\dot{\vartheta}}\left(-\frac{e v \|}{c R} \Psi_{n}-e \Phi_{n}\right) \exp [i(s \theta-n \chi)]
$$

Now we expand $I$ in a power series in $J-J_{r}$, where the subscript ' $r$ ' refers to the resonant torus, keeping the terms of $\bar{I}$ up to the second order and only the zeroth-order term of $\tilde{I}$ and using Eq. (37). This yields: 


$$
I=\bar{I}\left(J_{r}\right)+\left.\frac{\partial \bar{I}}{\partial J}\right|_{J-J_{r}}\left(J-J_{r}\right)+\left.\frac{1}{2} \frac{\partial^{2} \bar{I}}{\partial J^{2}}\right|_{J-J_{r}}\left(J-J_{r}\right)^{2}+\left.\Delta I\right|_{J-J_{r}} \exp (i \hat{\phi}) \text {. }
$$

The lines of level of $I$ represent orbits of the bounce-averaged motion in the presence of the perturbation in the vicinity of the resonance. They form a Chirikov island near the resonance. ${ }^{13}$ The separatrix of the island separates the orbits of the particles that are trapped with respect to the perturbation of the electromagnetic field (i.e., superbanana orbits) from the other orbits. This pattern agrees with the Poincaré maps obtained above. Note that similar approach was used to study the motion of the marginally trapped narrow-orbit particles during a sawtooth crash $^{11}$ and of locally trapped particles in stellarators. ${ }^{23}$

The equation (39) enables us to to evaluate the resonance width $\Delta J$ (i.e., the maximum superbanana orbit width) in terms of the amplitude of $\tilde{I}$ on the resonant drift surface, $\Delta I$. It follows from Eqs. (B9), (B10) that $\partial \bar{I} / \partial J=s / n-\omega_{\phi} / \omega_{b}$. Substituting this to Eq. (39), we obtain:

$$
\Delta J=2|\Delta I|^{1 / 2}\left|\frac{\partial}{\partial J} \frac{\omega_{\phi}}{\omega_{b}}\right|^{-1 / 2} .
$$

Calculation of the Chirikov island width from Eqs. (38), (40) requires only computation of several integrals along an unperturbed resonant trajectory and takes much less time than generating a Poincaré map. Comparison of $\Delta J$ calculated with these two methods shows a good agreement unless the perturbation amplitude is sufficiently large to cause stochastization of particle motion near the island separatrix [in the latter case, Eq. (40) gives a lower estimate for the island width]. In particular, when $\Delta r_{b} \ll r$ and $\omega_{\phi}=0$, Eq. (40) yields the superbanana width obtained in Ref. ${ }^{11}$.

In order to calculate the period of motion along a superbanana orbit, $\tau_{s b}$, we use Eqs. (35), (39). We find:

$$
\tau_{s b}=\frac{2 \pi}{n \omega_{b}}\left|\Delta I \frac{\partial}{\partial J} \frac{\omega_{\phi}}{\omega_{b}}\right|^{-1 / 2}
$$

In conclusion, we consider a numerical example. Using Eqs. (29), (30), (38), (40), we calculate the width of the resonances $1 / 3,1 / 2$ and $2 / 3$ for $1.4-\mathrm{MeV}$ alpha 
particles or $1.9-\mathrm{MeV}{ }^{3} \mathrm{He}$ ions in a JET-like tokamak. The results obtained in the assumption that $\Phi \neq 0, \Psi=0$ are shown in Fig. 6 . We see that the resonances overlap. The obtained picture is weakly sensitive to variation of the particle energy because location of the resonance curves on the $(L, P)$-plane almost does not depend on $\mathcal{E}$ (see Appendix A), and the resonance width depends on $\mathcal{E}$ rather weakly. Taking into account these facts and that $\Delta r_{b} \sim r_{s}$ in our calculations (because $\left.\Delta r_{b} \sim \nu R_{0}\right)$, we conclude that the resonant particles constitute an essential fraction of 'potatoes'.

Overlap of resonances is known to lead to stochastic diffusion. Stochastization was indeed observed in Poincaré maps corresponding to the regions of the overlapping resonances. This stochasticity is much more important than the stochasticity near the island separatrix (shown in Figs. 4, 5). The reason is that the stochasticity caused by the overlap requires less time to manifest itself and can occupy the whole sawtooth mixing region.

\section{Application to JET experiments}

It is of interest to apply our understanding of behavior of resonant and nonresonant particles to experiments with Ion Cyclotron Resonance Frequency (ICRF) heating on JET, where the bulk of ICRF-accelerated ${ }^{3}$ He ions was not sensitive to the crash, whereas a small population of these ions ('hot spot') was strongly redistributed. ${ }^{5}$ The estimated energies of observed ions were about $2 \mathrm{MeV}$, which well exceeded $\mathcal{E}_{\text {crit }}$. Thus, one can expect that the 'hot spot' was produced by resonant particles in contrast to the bulk of the fast ions.

In order to check this hypothesis, let us consider ions with $\mathcal{E}=2 \mathrm{MeV}$. We take the pitch angle of a hot-spot particle to be $100^{\circ}$ at $R-R_{0}=20 \mathrm{~cm} .{ }^{5}$ For a typical fast ion, we take $\lambda \equiv \mu B_{0} / \mathcal{E}=1$ and the banana tip radius $r_{t}=28 \mathrm{~cm}$. We obtain $L=-0.37, P=-0.085$ for the hot-spot ion and $L=0, P=1.1$ for the typical ion. Using Figs. 2 and 3, we conclude that the hot-spot ion is a poloidally trapped 
and toroidally untrapped one located in the region of the resonances $s=2, n=3$ and $s=1, n=2$, whereas the typical ion is outside of the region of resonances with $n=1 \div 3$.

More detailed information can be obtained from numerical simulations of motion of the ions during the crash. Calculations indicate that, in spite of the fact that the hot-spot ion is located close to the resonance $s=2, n=3$, the main role in its displacement belongs to the resonance $s=1, n=2$. The Poincaré maps demonstrating response of the hot-spot ion to a $m=n=2$ perturbation are presented in Fig. 7. We see from Fig. 7 (a) that the outer part of the orbit is essentially displaced outwards during the motion along a superbanana orbit. It follows from Fig. 7 (b) and Figs. 2, 3 that, first, the particle passes through the resonance $1 / 2$ and approaches the resonances $1 / 3$ and $2 / 3$ and, second, the orbit transforma-

tions $\mathrm{PT}^{+} \leftrightarrow \mathrm{T}$ take place (see the caption of Fig. 2 for notations). The period of the superbanana motion was of the order of the crash duration. When we added the $m=n=3$ harmonic, we observed stochastic motion of the hot-spot particle in a wide range of the parameter $P$ covering the resonances $1 / 3$ and $1 / 2$. Thus, resonant behavior of the hot-spot ions is a probable reason of their redistribution. However, to draw a definite conclusion, a detailed analysis is required.

\section{SUMMARY AND CONCLUSIONS}

We have investigated the conditions and effects of resonant wave-particle interaction during sawtooth crashes. The obtained results can be summarized as follows.

The frequency of the mode rotation and the equilibrium electric field are connected by the relationship $\omega=v_{E v} / r$. Due to this, the condition of resonant interaction between waves and particles can be presented in the form which does not depend on the mode frequency but depends on the electric field, the dependence on the electric field being associated only with influence of the electric field 
on the shape of particle orbits. In a special case of particles with narrow orbits, the resonance condition does not depend on $\omega$ and $\vec{E}$.

The resonant particles are more sensitive to the sawtooth crash than other particles. The reason is that the resonant particles, being trapped or marginally untrapped in the electromagnetic perturbation associated with a crash, have a perturbed orbit width which considerably exceeds the width of other orbits, see Fig. 5. Those particles which are trapped in the perturbation field move along superbanana orbits under influence of the crash. Motion of the particles with $\Delta r_{b} \geq r_{s}$ ('large potatoes') is similar to that of the particles with $\Delta r_{b} \ll r$ studied in Ref. ${ }^{11}$. However, the influence of resonances on the population of large potatoes is much greater. Only a narrow group of particles with $\Delta r_{b} \ll r$, namely, marginally trapped particles with $\kappa \approx 0.9$ are resonant. In contrast to this, there are many groups of resonant particles among 'potatoes' because the $s \neq 0$ resonances $\left(\omega_{\phi} / \omega_{b}=1 / 3,1 / 2,2 / 3\right)$ are possible for these particles. The mentioned resonances take place due to both the features of particle motion $\left(\omega_{b} \geq \omega_{\phi}\right)$ and the presence of the $n=2,3$ harmonics in the electromagnetic perturbation. Note, that the harmonics with $n=2,3$ have significant amplitudes even if the crash results from the $m=n=1$ instability. The dominant harmonic $(m=n=1)$ interacts resonantly only with those particles that are characterized by negligible toroidal precession.

The period of the superbanana motion is less or, at least, comparable to the crash duration. As a result, plateaus are formed on the radial profile of the fast ion distribution function near the resonant surfaces.

These results were obtained both numerically, by generating a Poincaré map, and analytically. In the analytical approach, a new adiabatic invariant given by Eqs. (31)-(34), was used. In addition, the frequencies of unperturbed motion of particles with arbitrary orbit width in the poloidal and toroidal directions were calculated, which may be of general interest, too.

The above described motion of resonant particles and the difference in behavior 
of resonant and non-resonant particles take place only when the particle energy is sufficiently large, $\mathcal{E}>\mathcal{E}_{\text {crit }}$, where $\mathcal{E}_{\text {crit }}$ is given by Eq. (1). Otherwise, the width of perturbed orbits is of the order of the sawtooth mixing radius, and all particles are strongly redistributed by sawtooth oscillations. $\mathcal{E}_{\text {crit }}$ was introduced for the first time in Ref. ${ }^{1}$. Here we have shown that $\mathcal{E}_{\text {crit }}$ does not depend on the mode rotation and the equilibrium electric field.

The particles having non-standard unperturbed orbits with small orbit width, e.g., 'small potatoes' with $\Delta r_{b} \ll r_{s}$, become standard orbit particles as a result of the crash-induced redistribution. That is why the $s \neq 0$ resonances are important only for particles with $\Delta r_{b}$ comparable to $r_{s}$. This yields additional restrictions for the particle energy. The first we obtain using the condition $\Delta r_{b}(\kappa=1) \geq r_{s}$, which can be written as $\mathcal{E}>\mathcal{E}_{1}$, where $\mathcal{E}_{1}$ is given by

$$
\mathcal{E}_{1}=\frac{M r_{s}^{3} \omega_{B}^{2}}{16 R}=10^{-2} \omega_{B} \tau_{c r} \mathcal{E}_{c r i t} \epsilon_{s}^{2}
$$

where $\epsilon_{s} \equiv r_{s} / R_{0}$. The second condition is $\mathcal{E}>\mathcal{E}_{2}$, where

$$
\mathcal{E}_{2}=8 \mathcal{E}_{1}
$$

Eq. (43) is determined from the condition $\Delta r_{b}\left(\kappa_{\text {min }}\right)=r_{s}$, with $\kappa_{\text {min }}$ satisfying the equation $\left.v_{\|}\right|_{r=r_{s}}=-\left.v_{D \vartheta}\right|_{r=r_{s}}$. When $\mathcal{E}>\mathcal{E}_{2}$, there are no particles with standard narrow orbits in the sawtooth mixing region.

It is convenient to show different behavior of various groups of particles in terms of the normalized particle energy and crash duration defined as follows: $\check{\mathcal{E}}=\rho^{2} /\left(\epsilon_{s} r_{s}^{2}\right), \check{\tau}_{c r}=\epsilon_{s}^{2} \omega_{B} \tau_{c r}$. This is done in Fig. 8 .

Because of the presence of the $s \neq 0$ resonances and the finite width of these resonances, the resonant particles may constitute an essential fraction of the high energy ions in the sawtooth mixing region. Furthermore, the resonances may overlap (see Fig. 6) so that all particles in a certain region of phase space may be resonant.

Different behavior of resonant and non-resonant ions seems to explain why a 
crash affects only a narrow group of ions ('hot spot') in ICRF heating experiments on JET. 5

\section{ACKNOWLEDGMENTS}

The research described in this publication was made possible in part by the Research Contract No. 8924 of the International Atomic Energy Agency and Award No. UP2-290 of the Government of Ukraine and the U.S. Civilian Research \& Development Foundation for the Independent States of the Former Soviet Union (CRDF). Any opinions, findings, conclusions or recommendations expressed in this material are those of the authors and do not necessarily reflect those of the Government of Ukraine and CRDF.

Two of the authors (Y.K. and Y.Y.) would like to acknowledge the hospitality of the Princeton Plasma Physics Laboratory.

\section{APPENDIX A: THE FREQUENCIES OF TOROIDAL AND POLOIDAL MOTION OF PARTICLES WITH ARBITRARY ORBIT WIDTH}

We assume that $q(r)=$ const, and the cross-sections of the flux surfaces are concentric ellipses of constant ellipticity $k$. In this case, the following equation of the particle orbit results from conservation of the magnetic moment, $\mu$, and the canonical angular momentum, $J_{T}$ :

$$
M^{2} v_{\|}^{2} R^{2}=2 M R\left(\mathcal{E} R-\mu B_{0} R_{0}\right)=\left(J_{T}+\frac{e}{c} \Psi_{p}\right)^{2}
$$

where $\Psi_{p}=F / q=B_{0}\left[k\left(R-R_{0}\right)^{2}+z^{2} / k\right] /(2 q)$ is the poloidal magnetic flux, $z$ is the vertical coordinate. Let us introduce the dimensionless parameters $L \equiv$ $\left(1-\mu B_{0} / \mathcal{E}\right) \nu^{-1}$ and $P \equiv-2 c J_{T} q /\left(k e B_{0} R_{0}^{2} \nu^{2}\right)$, where $\nu \equiv\left[2 q \rho /\left(k R_{0}\right)\right]^{2 / 3}, \rho \equiv$

$v / \omega_{B 0} \cdot{ }^{18,21}$ Then we can write Eq. (A1) in the following form:

$$
(L+\tilde{x})(1+\nu \tilde{x})=\left(\tilde{x}^{2}+\tilde{z}^{2}-P\right)^{2},
$$


where $\tilde{x} \equiv\left(R / R_{0}-1\right) / \nu, \tilde{z} \equiv z /\left(k R_{0} \nu\right), \nu$ being typically small. The equation (A2) is simplified if we take $\left|R-R_{0}\right| / R_{0} \ll 1$ :

$$
L+\tilde{x}=\left(\tilde{x}^{2}+\tilde{z}^{2}-P\right)^{2},
$$

We conclude from Eq. (A3) and the definition of $\tilde{x}$ and $\tilde{z}$ that variation of the particle energy at fixed $L$ and $P$ changes only the size of the orbit on the $(R, z)$ plane, the orbit shape being almost invariant.

At first, we analyze the number of orbits for given $L$ and $P$. For this purpose, we consider Eq. (A3) for $z=0$. This equation may have 0,2 or 4 real roots, which corresponds to presence of 0,1 and 2 orbits, respectively. The curves separating the regions with different number of the roots (i.e., different orbit topology) on the $(L, P)$-plane are characterized by coincidence of a pair of roots, whence we obtain the following parametric equations of the separating curves:

$$
P=\tilde{x}_{*}^{2}-\frac{1}{4 \tilde{x}_{*}}, \quad L=-\tilde{x}_{*}+\frac{1}{16 \tilde{x}_{*}^{2}},
$$

where $\tilde{x}_{*}$ equals to the pair of coinciding roots. A detailed analysis shows that the dashed curve in Fig. $2\left(\tilde{x}_{*}<-1 / 2\right)$ is the locus of the orbits with an $\mathrm{X}$-point (the pinch orbits), whereas the solid curves in Fig. $2\left(\tilde{x}_{*}>-1 / 2\right)$ are the loci of the orbits reduced to a point in the $(\tilde{x}, \tilde{z})$-plane (the stagnation orbits).

To accomplish the classification of orbits, we need to determine which of them belong to trapped or passing particles. The margin of toroidal trapping is given by the condition that $v_{\|}=0$ at $\tilde{z}=0$, which leads to $P=L^{2}$. The margin of poloidal trapping corresponds to passing of the orbit through the point $\tilde{x}=\tilde{z}=0$, whence $L=P^{2}$. Adding these two curves (dotted lines in Fig. 2), we arrive at the orbit classification presented in Fig. 2. Numerical calculations show that small but finite $\nu$ weakly changes the classification.

To calculate the frequencies of the particle motion, we proceed from the following expressions for the frequencies [cf. Eqs. (29), (30)]:

$$
\omega_{b}=2 \pi\left[\oint d \Psi_{p}\left(\dot{\Psi}_{p}\right)^{-1}\right]^{-1}
$$




$$
\omega_{\phi}=\frac{\omega_{b}}{2 \pi} \oint \frac{d \Psi_{p}}{\dot{\Psi}_{p}} \frac{v_{\|}}{R}
$$

$\dot{\Psi}_{p}$ can be found from Eq. (6). Transforming to the coordinates $\tilde{x}$ and $\tilde{z}$, we obtain:

$$
\omega_{b}=\frac{v \nu^{1 / 2}}{q R_{0}} F_{b}(L, P, \nu), \quad \omega_{\phi}=q \omega_{b} F_{\phi}(L, P, \nu),
$$

where

$$
\begin{gathered}
F_{b}=\pi\left[\oint d \tilde{\Psi}\left(\frac{1}{\tilde{z}}\right) \frac{1+\nu \tilde{x}}{1+L \nu+2 \nu \tilde{x}}\right]^{-1}, \\
F_{\phi}=\frac{1}{\pi} \oint d \tilde{\Psi} \frac{P-\tilde{\Psi}}{\tilde{z}(1+L \nu+2 \nu \tilde{x})(1+\nu \tilde{x})},
\end{gathered}
$$

$\tilde{\Psi} \equiv \tilde{x}^{2}+\tilde{z}^{2}$, and the integration is performed along an orbit defined by Eq. (A2). When $L \nu \ll 1$, the functions $F_{b}(L, P, \nu)$ and $F_{\phi}(L, P, \nu)$ weakly depend on $\nu$ (note that the functions are double-valued when there are two orbits for given $L, P$, and $\nu)$. This means, in particular, that the ratio $\omega_{\phi} / \omega_{b}$ determining resonance groups of particles weakly depends on $\mathcal{E}$ when the $L$ and $P$ are fixed and $L \lesssim 1$. This conclusion is supported by direct numerical calculations of $\omega_{\phi} / \omega_{b}$ using Eq. (6). The contour map of $\omega_{\phi} /\left(q \omega_{b}\right)=F_{\phi}(L, P, \nu)$ for $\nu=0.097$ is presented in Fig. 3.

The fact that $F_{b}$ and $F_{\phi}$ weakly depend on $\nu$ enables us to approximate them by their values at $\nu=0$. The latter can be found analytically in terms of $\tilde{x}_{1}$ and $\tilde{x}_{2}$, where $\tilde{x}_{1}$ and $\tilde{x}_{2}, \tilde{x}_{1}>\tilde{x}_{2}$, are the points of intersection of the orbit with the axis $\tilde{z}=0$. Indeed,

$$
\begin{gathered}
F_{b}=\frac{\pi}{2}\left[\int_{\tilde{x}_{2}^{2}}^{\tilde{x}_{1}^{2}} d \tilde{\Psi} Q^{-1 / 2}(\tilde{\Psi})\right]^{-1}, \\
F_{\phi}=\frac{2}{\pi} \int_{\tilde{x}_{2}^{2}}^{\tilde{x}_{1}^{2}} d \tilde{\Psi}(\tilde{\Psi}-P) Q^{-1 / 2}(\tilde{\Psi}),
\end{gathered}
$$

where $Q(\tilde{\Psi})=\tilde{\Psi}-\left[(\tilde{\Psi}-P)^{2}-L\right]^{2}$ is, $\tilde{z}^{2}$ expressed from Eq. (A3) as a function of $\tilde{\Psi}$. The integrals in Eqs. (A10), (A11) can be calculated analytically as elliptic integrals with arguments depending on the roots of $Q \cdot{ }^{24}$ One can see that the roots of $Q$ are the squares of the roots of Eq. (A3) for $z=0$. The equation $Q\left(\tilde{x}_{1}\right)=Q\left(\tilde{x}_{2}\right)=0$ 
can be solved for $P$ and $L$. Then all four roots of $Q$ are expressed through $\tilde{x}_{1}$ and $\tilde{x}_{2}$.

In particular, in the most important case when there are two real roots of $Q(\tilde{\Psi})$ [and, therefore, two real roots of Eq. (A3) for $z=0, \tilde{x}_{1}$ and $\tilde{x}_{2}$, we obtain:

$$
Q(\tilde{\Psi})=-\left(\tilde{\Psi}-\tilde{x}_{1}^{2}\right)\left(\tilde{\Psi}-\tilde{x}_{2}^{2}\right)\left[(\tilde{\Psi}-\eta)^{2}+\zeta\right]
$$

where $\eta=\left(\tilde{x}_{1}^{2}+\tilde{x}_{2}^{2}\right) / 2-1 / \xi, \zeta=\xi-\left(\tilde{x}_{1}^{2}-\tilde{x}_{2}^{2}\right)^{2} / 4$, and $\xi=\tilde{x}_{1}+\tilde{x}_{2}$. Using the integral tables, ${ }^{24}$ we find:

$$
\begin{gathered}
F_{b}=\frac{\pi}{4} A^{1 / 2} K(\kappa), \\
F_{\phi}=\frac{2}{\pi A^{1 / 2}}\left\{\xi^{-1} K(\kappa)+\left(\xi^{2}+\xi^{-1}+\xi A\right)\left[\Pi\left(\frac{\pi}{2}, \Delta, \kappa\right)-K(\kappa)\right]\right\},
\end{gathered}
$$

where $K$ and $\Pi$ are the complete elliptic integrals of the first and third kind, respectively,

$$
\begin{gathered}
A=\left(2 \tilde{x}_{1}+\xi^{-2}\right)^{1 / 2}\left(2 \tilde{x}_{2}+\xi^{-2}\right)^{1 / 2}, \\
\Delta=\frac{\left(\tilde{x}_{1}-\tilde{x}_{2}\right)^{2}}{A\left[\left(2 \tilde{x}_{1}+\xi^{-2}\right)^{1 / 2}+\left(2 \tilde{x}_{2}+\xi^{-2}\right)^{1 / 2}\right]}, \\
\kappa=\left\{\frac{1}{2}-\frac{1}{2 A}\left[\xi-\frac{1}{2}\left(\tilde{x}_{1}^{2}-\tilde{x}_{2}^{2}\right)^{2}+\xi^{-2}\right]\right\}^{1 / 2} .
\end{gathered}
$$

The case of small orbit width, $\Delta r_{b} \ll r$, corresponds to $P \gg 1$. One can show that $\tilde{x}_{1} \simeq \tilde{x}_{2} \simeq P^{1 / 2}, \tilde{x}_{1}^{2}-\tilde{x}_{2}^{2} \sim P^{1 / 4}, \tilde{x}_{1}-\tilde{x}_{2} \sim P^{-1 / 4}$ in this case. Then Eqs. (A13), (A14) and (A17) take in the main order in $P$ the form

$$
\begin{gathered}
F_{b}=\frac{\pi P^{1 / 4}}{2^{3 / 2} K(\kappa)}, \\
F_{\phi}=\frac{2 E(\kappa)-K(\kappa)}{2^{1 / 2} \pi P^{3 / 4}}, \\
\kappa=\frac{\tilde{x}_{1}^{2}-\tilde{x}_{2}^{2}}{2^{3 / 2} P^{1 / 4}},
\end{gathered}
$$


which agrees with the well known expressions for the precession and trapping parameter of a trapped particle with small orbit width:

$$
\begin{gathered}
\omega_{b}=\frac{\pi}{2^{3 / 2}} \frac{v \epsilon^{1 / 2}}{q R_{0} K(\kappa)}, \\
\omega_{\phi} / \omega_{b}=\frac{2^{1 / 2} \rho q^{2}}{\pi k r \epsilon^{1 / 2}}[2 E(\kappa)-K(\kappa)], \\
\kappa^{2}=\frac{\mathcal{E}-\mu B_{0}(1-\epsilon)}{2 \epsilon \mu B_{0}},
\end{gathered}
$$

where $\epsilon \equiv r / R_{0}$.

\section{APPENDIX B: RESONANCE ADIABATIC INVARIANT}

We proceed from the following Lagrangian of the guiding center motion: ${ }^{25}$

$$
\mathcal{L}=\frac{e}{c} \vec{A} \cdot \dot{\vec{r}}+\frac{M v_{\|}}{B} \vec{B} \cdot \dot{\vec{r}}+\frac{M c}{e} \mu \dot{\Theta}-\left(e \Phi+\mu B+\frac{M}{2} v_{\|}^{2}\right)
$$

where $\Theta$ is the gyrophase. As we are not interested in the evolution of the gyrophase, we will omit the third term, consider $\mu$ as a parameter and deal with a system with only two degrees of freedom.

We use the representation (4) for the electromagnetic field and choose the gauge

$$
A_{1}=0, \quad A_{2}=F(r), \quad A_{3}=-\Psi
$$

for the covariant components of $\vec{A}$. Analyzing the equations of motion (6), one can find that they do not change in the leading order if $\vec{B}$ is replaced by the toroidal field $B_{T}=B_{0} R_{0} \vec{e}^{3}$ everywhere except for the terms associated with $\vec{v}_{\|}$. For this reason, we retain the contribution of the poloidal magnetic field only in the first term of the Lagrangian (B1), which is responsible for the longitudinal motion. Taking the kinetic energy $\mathcal{E}=\mu B_{0} R_{0} / R+M v_{\|}^{2} / 2$ as an independent variable instead of $v_{\|}$, we arrive at the following Lagrangian:

$$
\mathcal{L}=\frac{e}{c} \vec{A} \cdot \dot{\vec{r}}+M v_{\|}(r, \vartheta, \mathcal{E}) R \dot{\varphi}-(\mathcal{E}+e \Phi)
$$


Note that Eqs. (6), (7) can be derived from Eq. (B3) as the corresponding EulerLagrange equations. Below we will also use the differential of the action associated with $\mathcal{L}$,

$$
\mathcal{L} d t=\frac{e}{c} \vec{A} \cdot d \vec{r}+M v_{\|}(r, \vartheta, \mathcal{E}) R d \varphi-(\mathcal{E}+e \Phi) d t
$$

As first, we consider the unperturbed motion of a particle in the magnetic field with $q=1, \Phi=\Psi=0$. In this case, it is expedient to use the coordinates $(r, \vartheta, \varphi)$ instead of $(r, \alpha, \varphi)$ in order to take advantage of the axial symmetry. Then the Lagrangian (B3) becomes:

$$
\mathcal{L}_{u}=\frac{e}{c} F \dot{\vartheta}+\left[-\frac{e}{c} F+M v_{\|}(r, \vartheta, \mathcal{E}) R\right] \dot{\varphi}-\mathcal{E}
$$

The time independence and the axial symmetry immediately lead to conservation of $\mathcal{E}$ and $J(r, \vartheta, \mathcal{E})=-e F / c+M v_{\|} R$, which determines the invariant tori (drift surfaces) of the unperturbed motion.

Now we will introduce the action-angle canonical variables of the unperturbed system.

To introduce the canonical angles, we use the property that their time derivatives are constant along a trajectory. In addition, we demand that the canonical angles, $\theta$ and $\phi$, changed by $2 \pi$ after one turn around the drift surface in the poloidal and toroidal direction, respectively. Then we arrive at the canonical angles $\theta$ and $\phi$ defined by Eqs. (28).

As usual, the corresponding actions are introduced as the integrals of the action differential

$$
\mathcal{L}_{u} d t=\frac{e}{c} F d \vartheta+\left[-\frac{e}{c} F+M v_{\|}(r, \vartheta, \mathcal{E}) R\right] d \varphi-\mathcal{E} d t
$$

along arbitrary loops encircling an invariant torus in the poloidal and toroidal directions. The toroidal action is just $J$. Performing integration around a drift surface at $\varphi=$ const, we can see that the poloidal action $J_{p}$ is the toroidal magnetic flux enclosed by the surface [up to the factor $e / c$, see Eq. (33)]. For a narrow-orbit 
circulating particle, $J_{p}$ is just $(e / c) F$. For a narrow-orbit trapped particle, $J_{p}$ coincides with the longitudinal adiabatic invariant (the easiest way to see this is to direct the integration contour along a magnetic field line).

To show that the coordinates $\left(J_{p}, J, \theta, \phi\right)$ are indeed canonical, it is sufficient to prove that the Lagrangian

$$
\mathcal{L}_{u}^{*}=J_{p} \dot{\theta}+J \dot{\phi}-\mathcal{E}
$$

is equivalent to $\mathcal{L}_{u}$, i.e., the difference $\mathcal{L}_{u}^{*} d t-\mathcal{L}_{u} d t$ is the full differential of certain function of $t, \vec{r}$, and $\mathcal{E}$. This calculation is straightforward and, therefore, not presented here. Due to equivalence, we will not distinguish between $\mathcal{L}_{u}$ and $\mathcal{L}_{u}^{*}$ below.

To study the motion near the resonance $\omega_{\phi} / \omega_{b}=s / n$, we introduce new canonical angles associated with the resonance, $\hat{\phi}=n \phi-s \theta$ and $\hat{\theta}=\theta / n$. Then the Lagrangian (B7) takes the following form:

$$
\mathcal{L}_{u}=\hat{J}_{p} \dot{\hat{\theta}}+\hat{J} \dot{\hat{\phi}}-\mathcal{E}
$$

where $\hat{J}_{p} \equiv n J_{p}+s J, \hat{J} \equiv J / n$. The Euler-Lagrange equations resulting from Eq. (B8) are as follows:

$$
\begin{gathered}
\dot{\hat{\theta}}=\left(\frac{\partial \hat{J}_{p}}{\partial \mathcal{E}}\right)^{-1}=\omega_{b}(\mathcal{E}, \hat{J}) / n, \\
\dot{\hat{\phi}}=-\left(\frac{\partial \hat{J}_{p}}{\partial \mathcal{E}}\right)^{-1} \frac{\partial \hat{J}_{p}}{\partial \hat{J}}=n \omega_{\phi}(\mathcal{E}, \hat{J})-s \omega_{b}(\mathcal{E}, \hat{J}), \\
\dot{\hat{J}}=\dot{\hat{J}}_{p}=0 .
\end{gathered}
$$

Note that we treat $\hat{J}_{p}$ as a function of $\hat{J}$ and $\mathcal{E}$, which are considered as independent variables together with $\hat{\theta}$ and $\hat{\phi}$. If we considered $\mathcal{E}$ as a function of $\hat{J}_{p}$ and $\hat{J}$, we would obtain the usual Hamilton's equations.

An immediate consequence of Eq. (B10) is that 


$$
\left.\frac{\partial \hat{J}_{p}}{\partial \hat{J}}\right|_{\hat{J}_{=\hat{J}_{r}(\mathcal{E})}}=0
$$

where $\hat{J}_{r}(\mathcal{E})$ is the value of $\hat{J}$ at the resonant drift surface of the resonance $\omega_{\phi} / \omega_{b}=$ $s / n$.

Now we proceed to study the particle motion in the presence of a perturbation. Our idea is to use the fact that 'detuning' of a particle from the resonance is expected to be small when the perturbation is small. Having this in mind, we consider $\mathcal{L}_{u}$ as a sum of two components of different orders. The first of them, which is responsible for the resonance motion, is defined as

$$
\mathcal{L}^{(0)}=\hat{J}_{p}^{(0)}(\mathcal{E}) \dot{\hat{\theta}}+\hat{J} \dot{\hat{\phi}}-\mathcal{E}
$$

where $\hat{J}_{p}^{(0)}(\mathcal{E}) \equiv \hat{J}_{p}\left(\mathcal{E}, \hat{J}_{r}(\mathcal{E})\right)$. The Euler-Lagrange equations resulting from Eq. (B13) describe bounce motion in resonance with the wave:

$$
\begin{gathered}
\dot{\hat{\theta}}=\left(\frac{\partial \hat{J}_{p}^{(0)}}{\partial \mathcal{E}}\right)^{-1}=\omega_{0}(\mathcal{E}) \equiv \omega_{b}\left(\mathcal{E}, \hat{J}_{r}\right) / n \\
\dot{\hat{\phi}}=0
\end{gathered}
$$

where we have taken into account Eq. (B12). We will assume that the residual $\hat{J}_{p}^{(1)} \equiv \hat{J}_{p}(\mathcal{E}, \hat{J})-\hat{J}_{p}^{(0)}(\mathcal{E})$ responsible for 'detuning' from the resonance $(\dot{\hat{\phi}} \neq 0)$ is of the order of $\varepsilon$, which is the ordering parameter indicating the order of magnitude of various terms (we will set $\varepsilon=1$ to obtain physically meaningful results). The same order is attributed to the terms that include $\Phi$ and $\Psi$ and are responsible for the perturbation. Then the Lagrangian (B3) can be written as follows:

$$
\mathcal{L}=\mathcal{L}^{(0)}+\varepsilon\left(\hat{J}_{p}^{(1)}(\mathcal{E}) \dot{\hat{\theta}}-\frac{e}{c} \Psi \dot{\varphi}-e \Phi\right)
$$

For the following calculations, it will be convenient to introduce the vector of variables $\vec{z}=\left(z_{1}, z_{2}, z_{3}, z_{4}\right) \equiv(\hat{\theta}, \hat{\phi}, \mathcal{E}, \hat{J})$. Expressing $\dot{\varphi}$ through $\dot{\vec{z}}$, we can rewrite Eq. (B16) as follows:

$$
\mathcal{L}=\gamma_{j}(\vec{z}) \dot{z}_{j}-H(\vec{z})
$$


where $H=H^{(0)}+\varepsilon H^{(1)}=\mathcal{E}+\varepsilon e \Phi, \gamma_{j}=\gamma_{j}^{(0)}+\varepsilon \gamma_{j}^{(1)}$. Here and below we imply summation over repeating subscripts. The zeroth-order contributions to $\vec{\gamma}$ are $\gamma_{1}^{(0)}=\hat{J}_{p}^{(0)}(\mathcal{E}), \gamma_{2}^{(0)}=\hat{J}, \gamma_{3}^{(0)}=\gamma_{4}^{(0)}=0$. As we will see below, it is not necessary to know the components of $\vec{\gamma}^{(1)}$, although they can be easily established by expressing $d \varphi$ in terms of $d \vec{z}$.

Now we assume that the particle motion near the resonance is not stochastic and can be characterized by an adiabatic invariant. To find the invariant, we apply the following method. ${ }^{26}$ We assume that the invariant $(I)$ can be found as a power series in $\varepsilon, I(\vec{z})=I^{(0)}(\vec{z})+\varepsilon I^{(1)}(\vec{z})+\ldots$, and is a single-valued function periodic along the angle variables $\left(z_{1}\right.$ and $\left.z_{2}\right)$. We restrict ourselves to the case of steadystate perturbations and, thus, do not suppose $I$ to depend on time explicitly. Now we will solve the equation $d I / d t=0$ order by order.

At the lowest order, only $\dot{\hat{\theta}}^{(0)}=\omega_{0}$ is non-zero [see Eqs. (B11), (B14), (B15)], which leads to $\partial I^{(0)} / \partial \hat{\theta}=0$. The next order gives

$$
\frac{\partial I^{(1)}}{\partial \hat{\theta}} \omega_{0}+\frac{\partial I^{(0)}}{\partial z_{i}} \dot{z}_{i}^{(1)}=0
$$

To exclude $I^{(1)}$, we integrate Eq. (B18) along $\hat{\theta}$ over one period and use periodicity of $I^{(1)}$. We obtain:

$$
\frac{\partial I^{(0)}}{\partial z_{i}} \oint d \hat{\theta} \dot{z}_{i}^{(1)}=0
$$

¿From this point, we will omit the superscript ' $(0)$ ' at $I$.

The Euler-Lagrange equations following from Eq. (B17) can be written in the form $^{25}$

$$
\dot{z}_{i}=\Pi_{i j} \frac{\partial H}{\partial z_{j}}
$$

where $\Pi_{i j}=\left\{z_{i}, z_{j}\right\}$ are the Poisson brackets of the variables, which are the inverse of the Lagrange brackets:

$$
\Pi_{i j} w_{j k}=\delta_{i k}
$$


where $\delta_{i k}$ is the Kronecker symbol. The Lagrange brackets $w_{i j}=\left[z_{i}, z_{j}\right]$ can be found from the coefficients of the Lagrangian:

$$
w_{i j}=\frac{\partial \gamma_{j}}{\partial z_{i}}-\frac{\partial \gamma_{i}}{\partial z_{j}}
$$

Expanding Eqs. (B20)-(B22) to the first order in $\varepsilon$ to find $\dot{z}_{i}^{(1)}$, we transform Eq. (B19) to

$$
\frac{\partial I}{\partial z_{i}} \Pi_{i l}^{(0)} \oint d \hat{\theta}\left[\frac{\partial H^{(1)}}{\partial z_{l}}+\left(\frac{\partial \gamma_{l}^{(1)}}{\partial z_{k}}-\frac{\partial \gamma_{k}^{(1)}}{\partial z_{l}}\right) \Pi_{k j}^{(0)} \frac{\partial H^{(0)}}{\partial z_{j}}\right]=0 .
$$

One can find from Eqs. (B13), (B21) and (B22) that the only non-zero components of $\Pi_{i j}^{(0)}$ are

$$
\Pi_{13}^{(0)}=-\Pi_{31}^{(0)}=\omega_{0}, \quad \Pi_{24}^{(0)}=-\Pi_{42}^{(0)}=1
$$

Substituting these values for $\Pi_{i j}^{(0)}$ and $z_{3}$ for $H^{(0)}$, we obtain the final equation for $I:$

$$
\frac{\partial I}{\partial \hat{\phi}} \oint d \hat{\theta}\left(\frac{\partial H^{(1)}}{\partial \hat{J}}-\omega_{0} \frac{\partial \gamma_{1}^{(1)}}{\partial \hat{J}}\right)-\frac{\partial I}{\partial \hat{J}} \oint d \hat{\theta}\left(\frac{\partial H^{(1)}}{\partial \hat{\phi}}-\omega_{0} \frac{\partial \gamma_{1}^{(1)}}{\partial \hat{\phi}}\right)=0 .
$$

The general solution of this equation is an arbitrary function of $\mathcal{E}$ and

$$
\frac{1}{2 \pi} \oint d \hat{\theta}\left(\omega_{0} \gamma_{1}^{(1)}-H^{(1)}\right)
$$

We choose $I$ as follows:

$$
I=\frac{1}{n}\left[\hat{J}_{p}^{(0)}+\frac{1}{2 \pi} \oint d \hat{\theta}\left(\gamma_{1}^{(1)}-\omega_{0}^{-1}(\mathcal{E}) H^{(1)}\right)\right]
$$

$\operatorname{As} \gamma_{1}^{(0)}=\hat{J}_{p}^{(0)}$

$$
I=\frac{1}{2 \pi n}\left(\oint d \vec{z} \cdot \vec{\gamma}-\oint d \hat{\theta} \omega_{0}^{-1} e \Phi\right)
$$

where $\varepsilon$ in $\vec{\gamma}$ is set to unity. However, the differential of the action

$$
\mathcal{L} d t=\vec{\gamma} \cdot d \vec{z}-H d t
$$

with $\varepsilon=1$ is equivalent to Eq. (B4) up to a coordinate transformation. Thus, substituting Eq. (B4) for $d \vec{z} \cdot \vec{\gamma}$ in Eq. (B28) does not change $I$. We obtain: 


$$
I=\frac{1}{2 \pi n} \oint\left(\frac{e}{c} F d \vartheta+J d \varphi-\frac{e}{c} \Psi d \varphi-\omega_{0}^{-1} e \Phi d \hat{\theta}\right)
$$

Finally, we recall that the integration contour makes $n$ turns around the drift surface in the poloidal direction and $s$ turns in the toroidal one. The first two terms in Eq. (B30) evidently result in $\bar{I}$ given by Eq. (32). If we assume that the perturbation potentials $\Phi$ and $\Psi$ consist only of harmonics with the toroidal wavenumber $n$, we can replace the integral over $n$ poloidal turns by the integral over one turn multiplied by $n$. Then the last two terms of Eq. (B30) yield Eq. (34) if we neglect the deviation of $\omega_{b}$ and $\omega_{\phi}$ from their values on the resonance surface. In the general case, when the perturbation is not periodic in $\varphi$ with the period $2 \pi / n$, the integration should be done over the closed loop $\hat{\phi}=$ const, making $n$ poloidal turns. 


\section{REFERENCES}

${ }^{1}$ Ya. I. Kolesnichenko and Yu. V. Yakovenko, Report at the IV International Atomic Energy Agency Technical Committee Meeting on Alpha Particles in Fusion Research (Princeton, April 1995); Nucl. Fusion 36, 159 (1996).

${ }^{2}$ F. B. Marcus, J. M. Adams, A. D. Cheetham, S. Conroy, W. G. F. Core, O. N. Jarvis, M. J. Loughlin, G. Sadler, P. Smeulders, P. van Belle, and N. Watkins, Plasma Phys. Controlled Fusion 33, 277 (1991).

${ }^{3}$ F. B. Marcus, J. M. Adams, B. Balet, D. S. Bond, S. W. Conroy, P. J. A. Howarth, O. N. Jarvis, M. J. Loughlin, G. J. Sadler, P. Smeulders, and N. Watkins, Nucl. Fusion 33, 1325 (1993).

${ }^{4}$ M. P. Petrov, R. V. Budny, H. H. Duong, R. K. Fisher, N. N. Gorelenkov, J. M. McChesney, D. K. Mansfield, S. S. Medley, P. B. Parks, M. H. Redi, and A. L. Roquemore, Nucl. Fusion 35, 1437 (1995).

${ }^{5}$ O. N. Jarvis, J. M. Adams, P. J. A. Howarth, F. B. Marcus, E. Righi, G. J. Sadler, D. F. H. Start, P. van Belle, C. D. Warrick, and N. Watkins, Nucl. Fusion 36, $1513(1996)$.

${ }^{6}$ R. C. Stratton, R. J. Fonck, G. R. McKee, R. V. Budny, Z. Chang, F. Wising, and A. Ödblom, Nucl. Fusion 36, 1586 (1996).

${ }^{7}$ D. J. Grove and D. M. Meade, Nucl. Fusion 25, 1167 (1985).

${ }^{8}$ P. H. Rebut, R. J. Bickerton, and B. E. Keen, Nucl. Fusion 25, 1011 (1985).

${ }^{9}$ Ya. I. Kolesnichenko and Yu. V. Yakovenko, Nucl. Fusion 32, 449 (1992).

${ }^{10}$ Ya. I. Kolesnichenko, V. V. Lutsenko, Yu. V. Yakovenko, and G. Kamelander, Phys. Plasmas 4, 2544 (1997).

${ }^{11}$ Ya. I. Kolesnichenko, V. V. Lutsenko, and Yu. V. Yakovenko, "Superbanana orbits and redistribution of marginally trapped fast ions during sawtooth crashes", 
Preprint PMI 3457, École Polytechnique, Palaiseau (1997); to appear in Phys. Plasmas.

${ }^{12}$ Yi Zhao, R. B. White, Phys. Plasmas 4, 1103 (1997).

${ }^{13}$ B. V. Chirikov, Phys. Reports 52, 265 (1979).

${ }^{14}$ JET Team, in 11th International Atomic Energy Agency Conference on Plasma Physics and Controlled Nuclear Fusion Research, Kyoto, 1986 (International Atomic Energy Agency, Vienna, 1987), Vol. 1, p. 449.

${ }^{15}$ F. Romanelli, L. Chen, and R. B. White, Nucl. Fusion 31, 631 (1991).

16 J. A. Rome, Y-K. M. Peng, Nucl. Fusion 19, 1193 (1979).

${ }^{17}$ C. T. Hsu and D. J. Sigmar, Phys. Fluids B 4, 1492 (1992).

${ }^{18}$ F. Porcelli, L.-G. Eriksson, and H. L. Berk, in 21st European Physical Society Conference on Controlled Fusion and Plasma Physics, Montpellier, 1994 (The European Physical Society, Petit-Lancy, 1994).

${ }^{19}$ Ya. I. Kolesnichenko, M. Lisak, V. V. Lutsenko, and F. Wising, Plasma Phys. Controlled Fusion 37, 363 (1995).

${ }^{20}$ C. M. Doloc and G. Martin, Nucl. Fusion 35, 1543 (1995).

${ }^{21}$ Yu. V. Yakovenko, V. V. Lutsenko, and Ya. I. Kolesnichenko, "On Particles with Non-Standard Orbits in Tokamaks", in 5th International Atomic Energy Agency Technical Committee Meeting on Alpha Particles in Fusion Research, Abingdon, 1997, to be published.

${ }^{22}$ A. N. Kaufman, Phys. Fluids 15, 1063 (1972).

${ }^{23}$ A. A. Galeev and R. Z. Sagdeev, in Problems of Plasma Theory (Consultants Bureau, New York, 1979), Vol. 7, p. 257.

${ }^{24}$ W. Gröbner, N. Hofreiter, Integraltafel. Erster Teil. Unbestimmte Integrale 
(Springer-Verlag, 1957) [in German].

${ }^{25}$ R. G. Littlejohn, J. Plasma Phys. 29, 111 (1983).

${ }^{26}$ J. R. Cary, C. L. Hedrick, J. S. Tolliver, Phys. Fluids 31, 1586 (1988). 


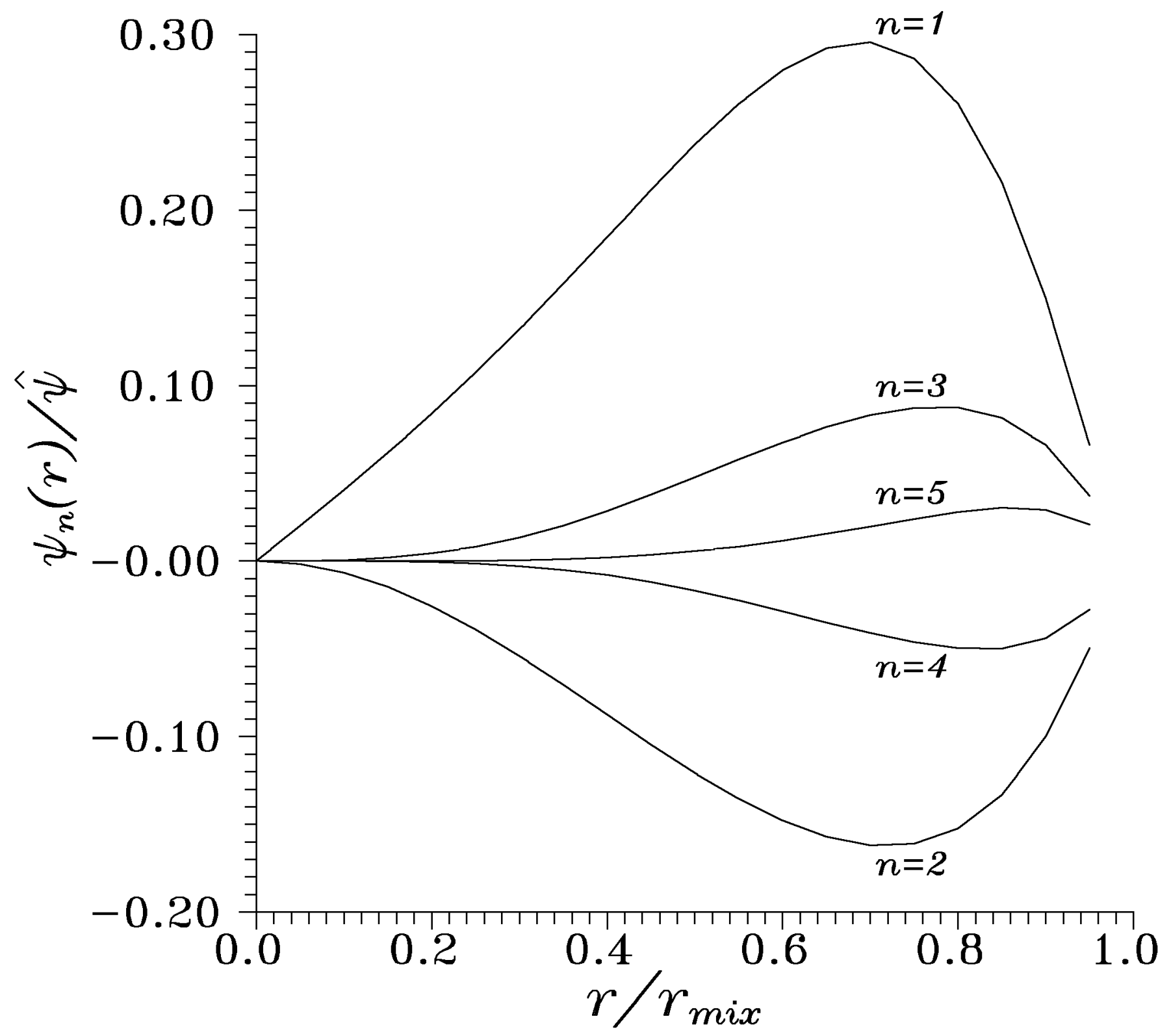




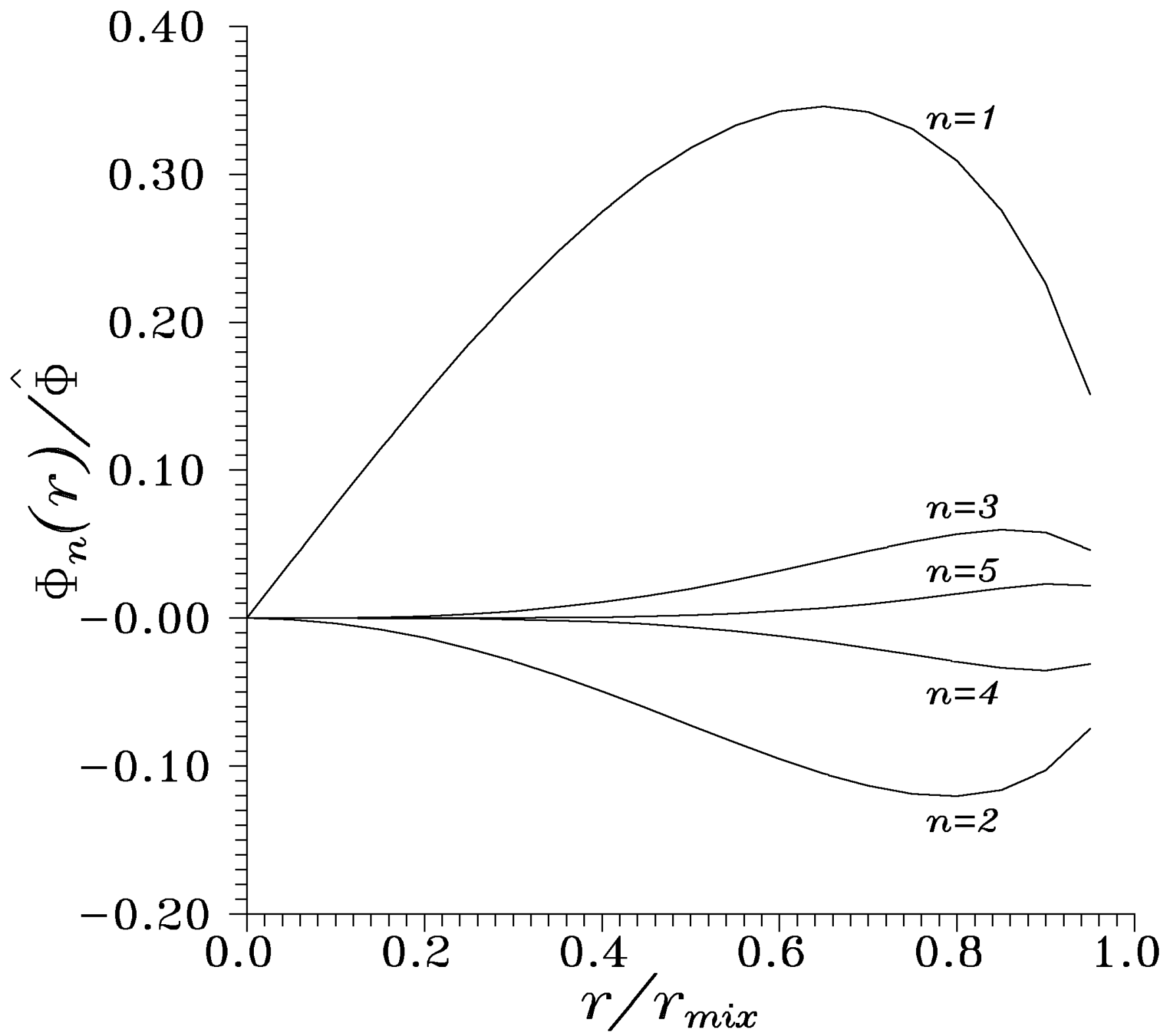

FIG. 1. Harmonics of perturbed electromagnetic field at the end of reconnection in the crash model of Ref. ${ }^{1}$. (a), the helical magnetic flux; (b), the potential of the electric field. $\hat{\Psi} \equiv k B_{0} r_{m i x}^{2}\left(q_{0}^{-1}-1\right) / 8$ and $\hat{\Phi} \equiv k B_{0} r_{m i x}^{2} /\left(c \tau_{c r}\right)$ are characteristic magnitudes of $\Psi$ and $\Phi$, respectively, $k$ is the cross-section ellipticity, $r_{m i x}$ is the sawtooth mixing radius. 


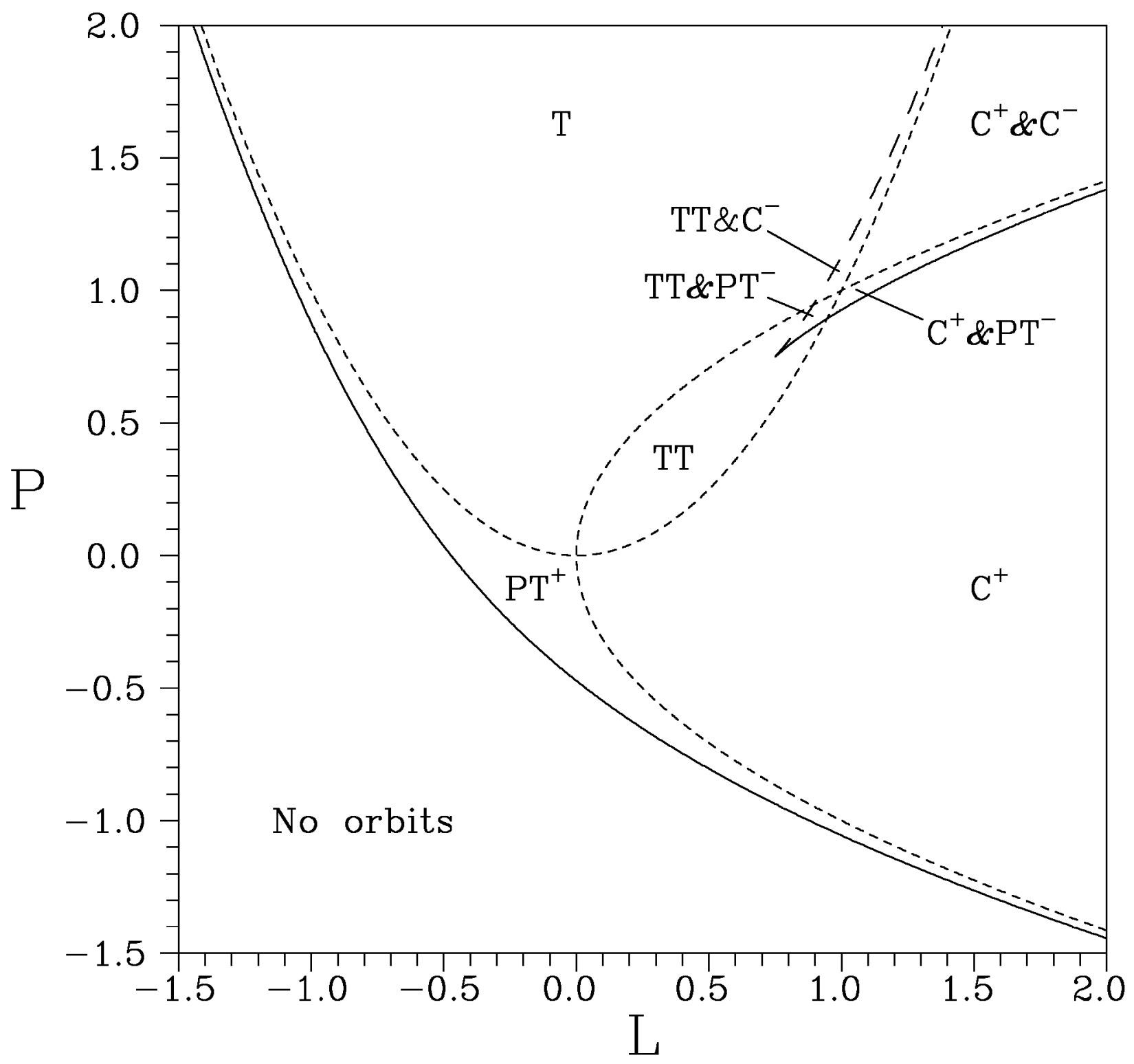

FIG. 2. Classification of particle orbits. Notations: $P \equiv-2 c J q /\left(k e B_{0} R_{0}^{2} \nu^{2}\right)$; $L \equiv\left(1-\mu B_{0} / \mathcal{E}\right) \nu^{-1} ; \nu \equiv\left[2 q \rho /\left(k R_{0}\right)\right]^{2 / 3} ; \mathrm{C}$, circulating; PT, poloidally trapped; TT, toroidally trapped; T, trapped (both poloidally and toroidally); superscripts '+' and '-' refer to $\operatorname{sgn} v_{\|} . P \gg 1$ corresponds to standard orbits with $\Delta r_{b} \ll r$. 


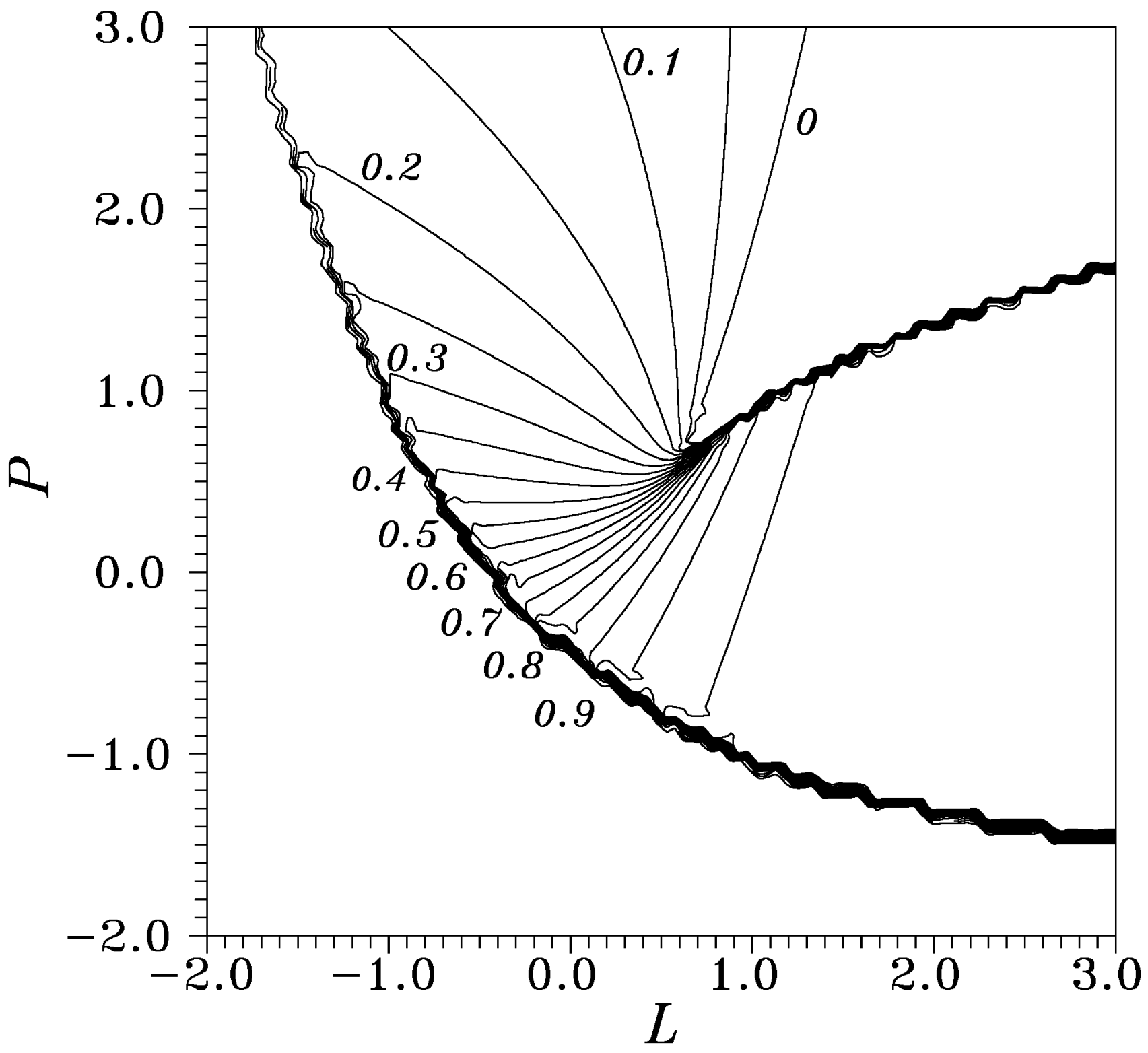

FIG. 3. Level contours of $\omega_{\phi} /\left(q \omega_{b}\right)$ for $\nu=0.11$. 


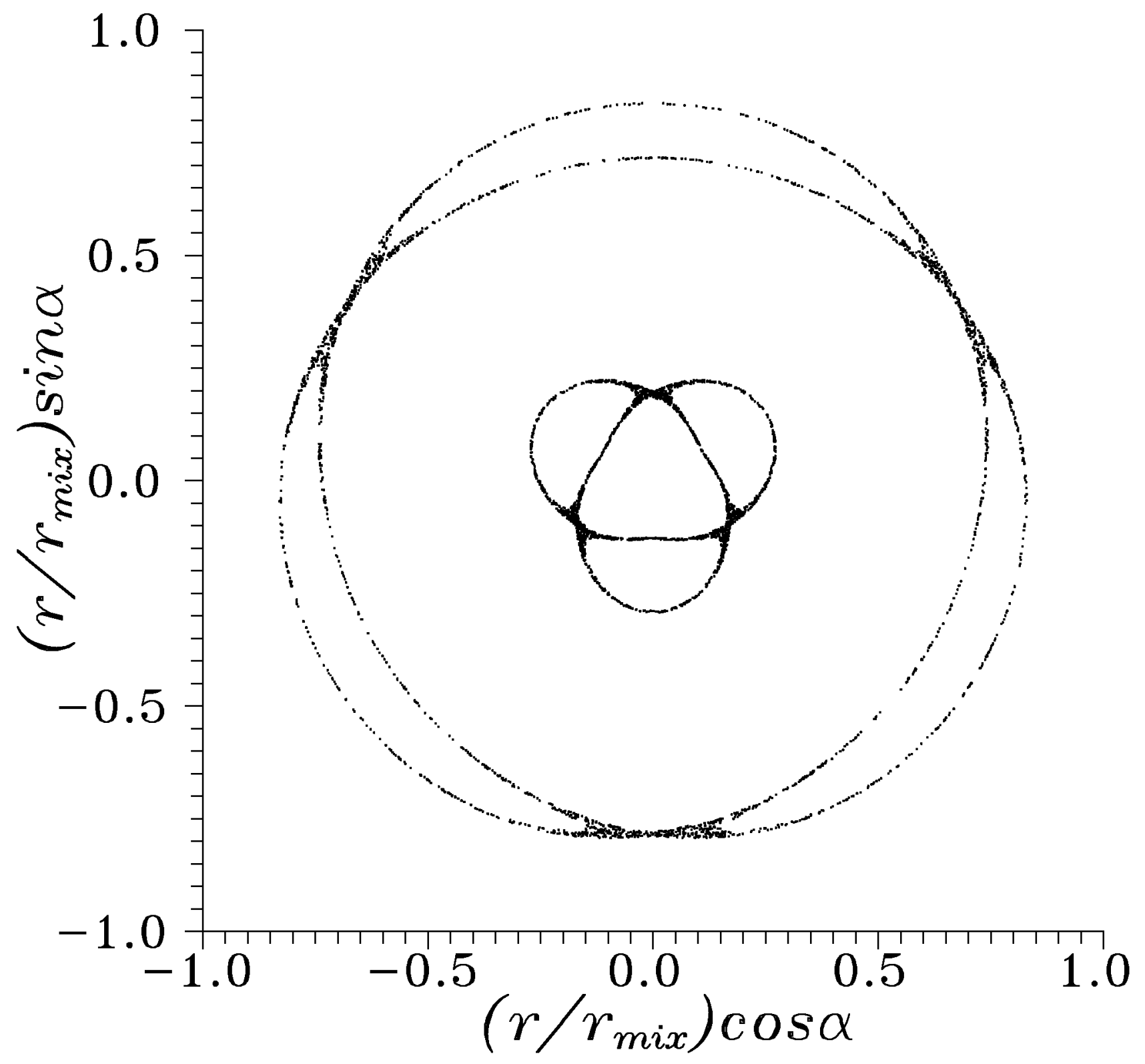




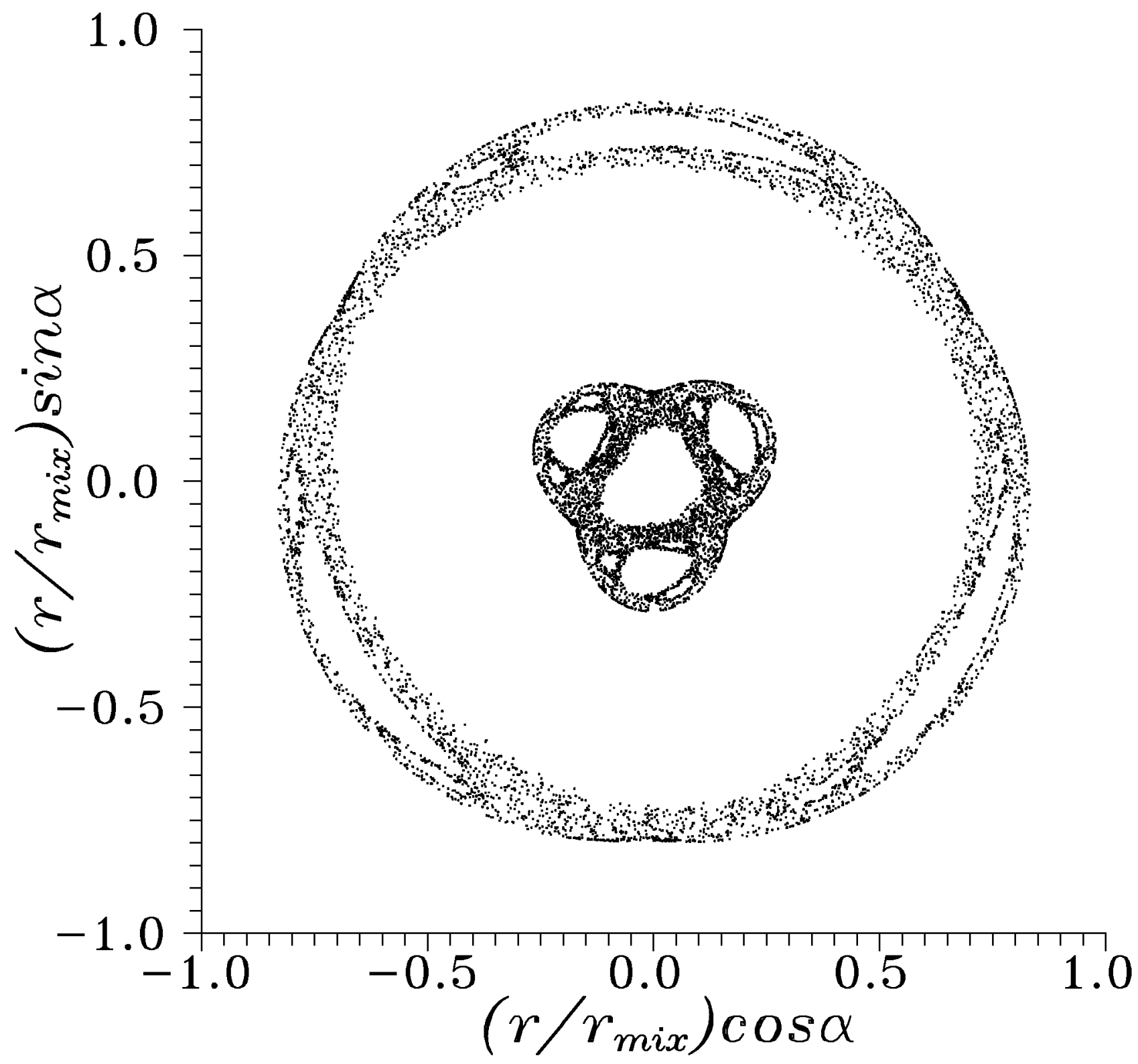




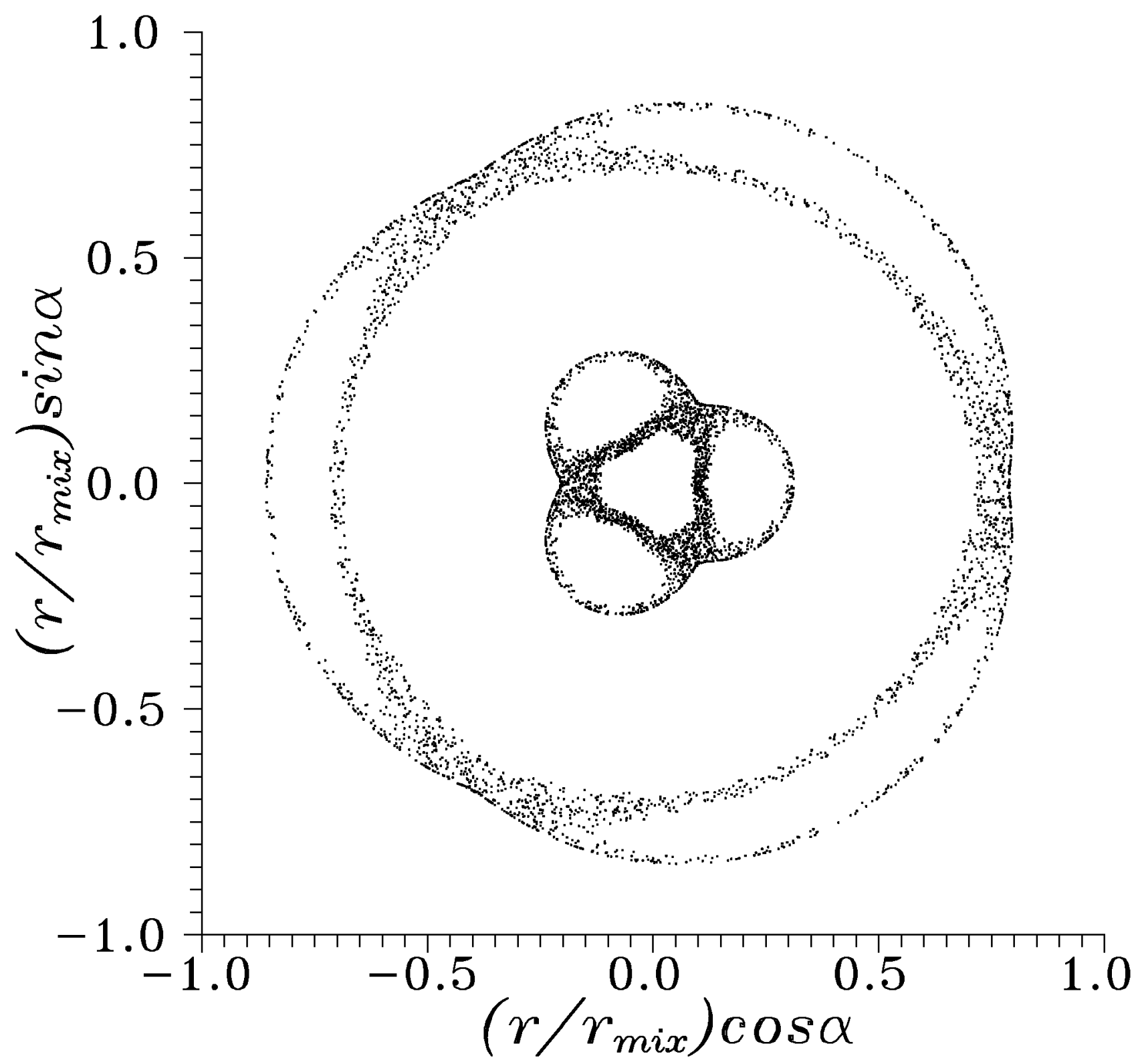

FIG. 4. Poincaré map of motion of fast ions in the presence of helical perturbations of the electromagnetic field. Used parameters: $\mathcal{E}=1.4 \mathrm{MeV}, \lambda \equiv \mu B_{0} / \mathcal{E}=1.007$, $B_{0}=3.5 \mathrm{~T}, R_{0}=3.0 \mathrm{~m}, \tau_{c r}=10^{-4} \mathrm{~s}, r_{\text {mix }}=0.4 \mathrm{~m}, k=1.5$. (a) single electrostatic harmonic, $\Phi_{3 \max }=0.1 \hat{\Phi}, \Phi_{2 \max }=\Psi_{2 \max }=\Psi_{3 \max }=0$; (b) superposition of two electrostatic harmonics, $\Phi_{3 \max }=0.1 \hat{\Phi}, \Phi_{2 \max }=0.025 \hat{\Phi}, \Psi_{2 \max }=\Psi_{3 \max }=0 ;$ (c) single magnetic harmonic, $\Psi_{3 \max }=0.1 \hat{\Psi}, \Psi_{2 \max }=\Phi_{2 \max }=\Phi_{3 \max }=0$. 


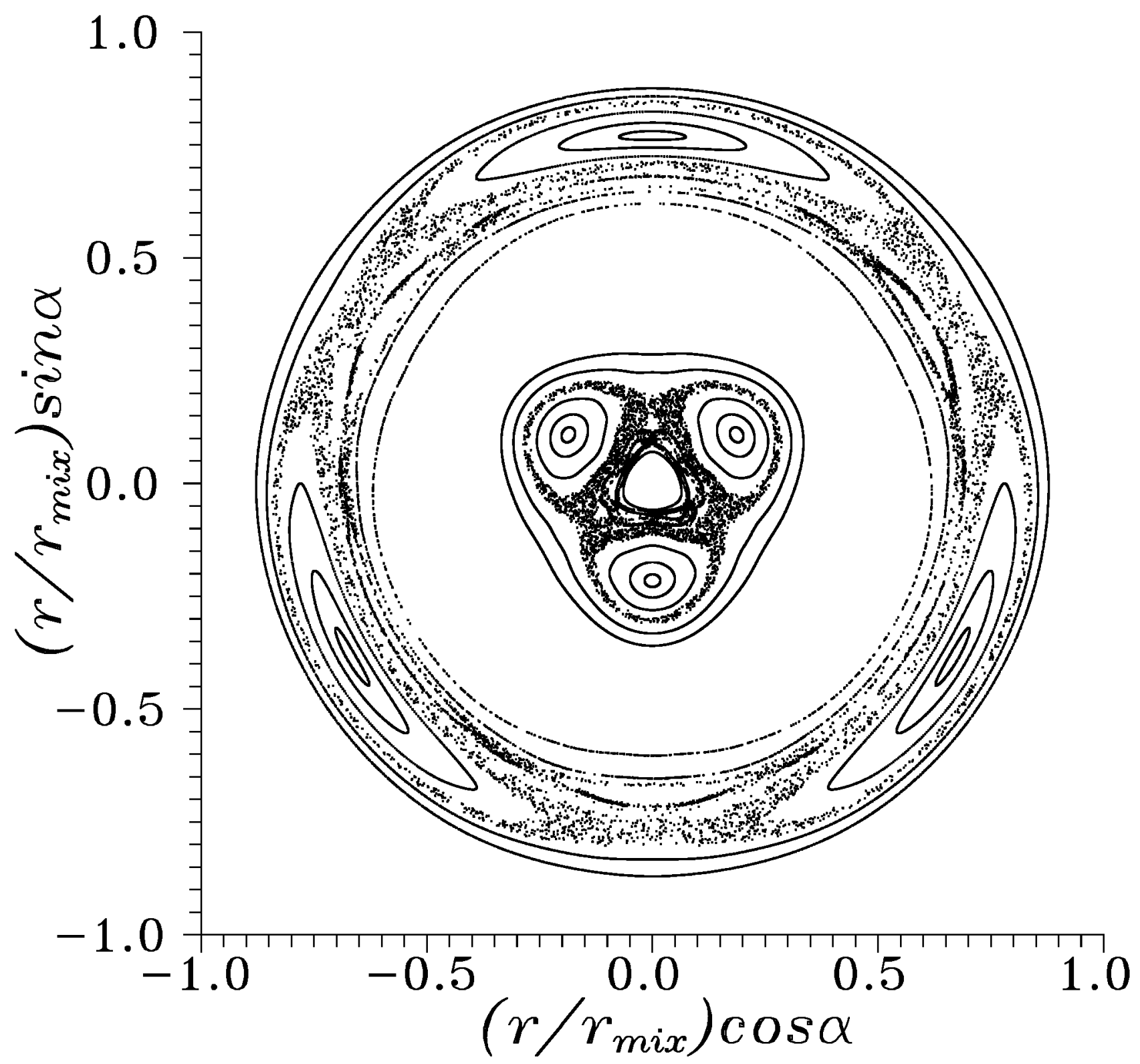

FIG. 5. Poincaré map showing several superbanana and passing orbits and a stochastic region in the presence of the perturbation with $\Phi_{3 \max }=0.15 \hat{\Phi}$, $\Phi_{2 \max }=\Psi_{2 \max }=\Psi_{3 \max }=0$. The used parameters are the same as in Fig. 4. 


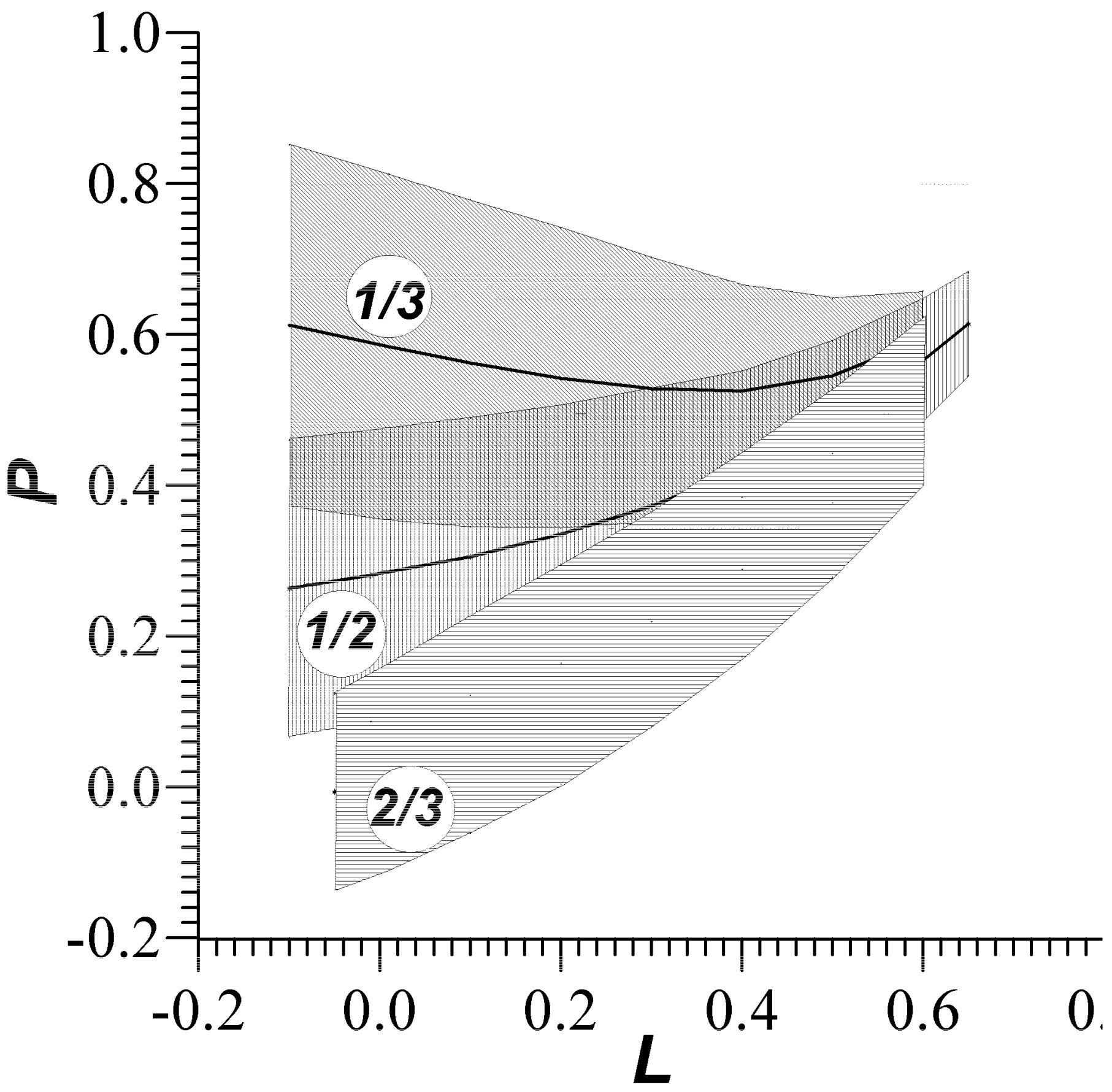

FIG. 6. Regions of resonances $\omega_{\phi} / \omega_{b}=s / n=1 / 3,1 / 2,2 / 3$ for $1.4-\mathrm{MeV} \alpha$-particles, $\Phi_{3 \max }=\Phi_{2 \max }=0.1 \hat{\Phi}$, and the same tokamak parameters as in Fig. 4. 


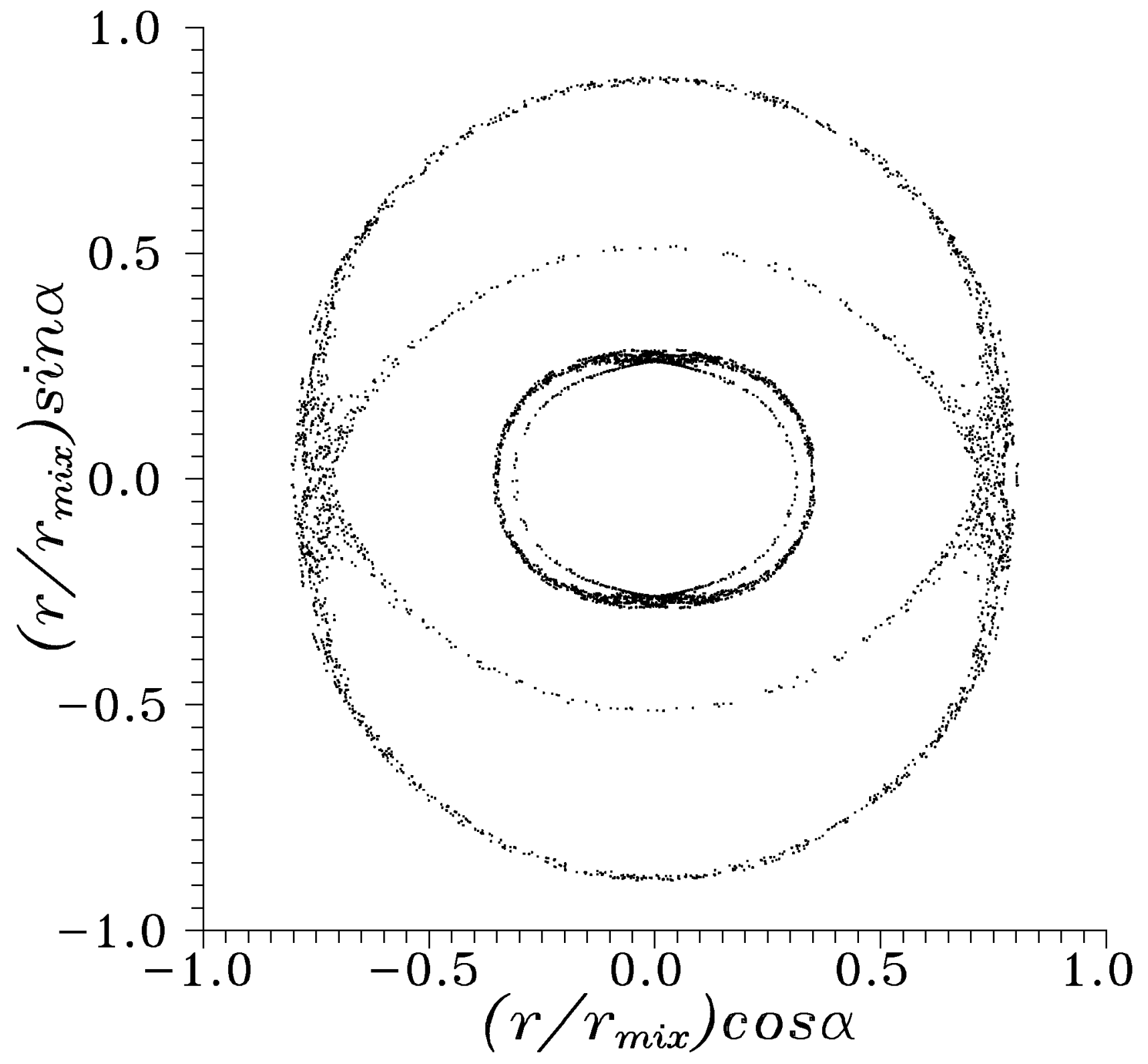




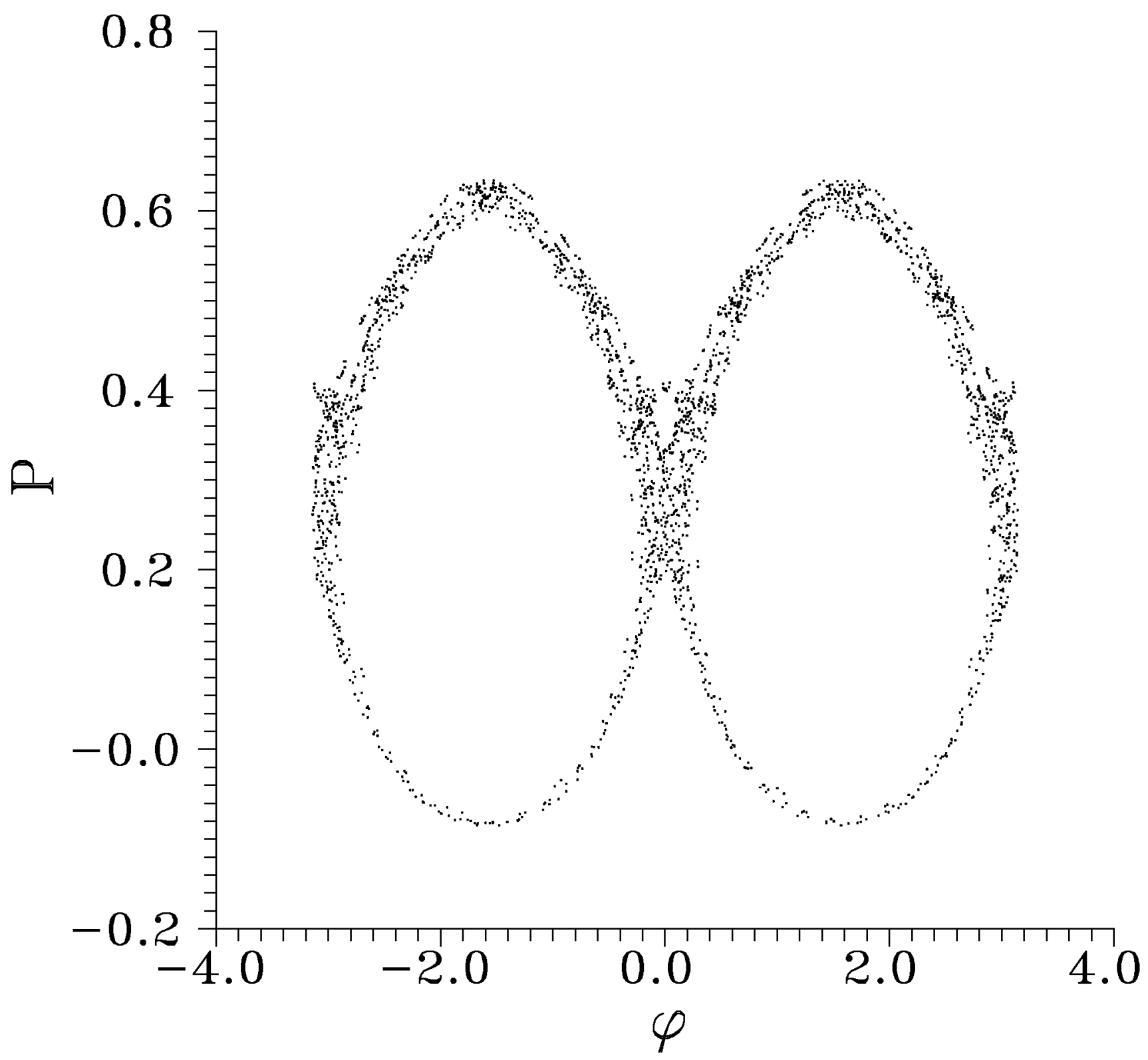

FIG. 7. Poincaré maps of motion of an ion relevant to the "hot spot'" under the $m=n=2$ perturbation, $\Phi_{2 \max }=0.32 \hat{\Phi}$. 


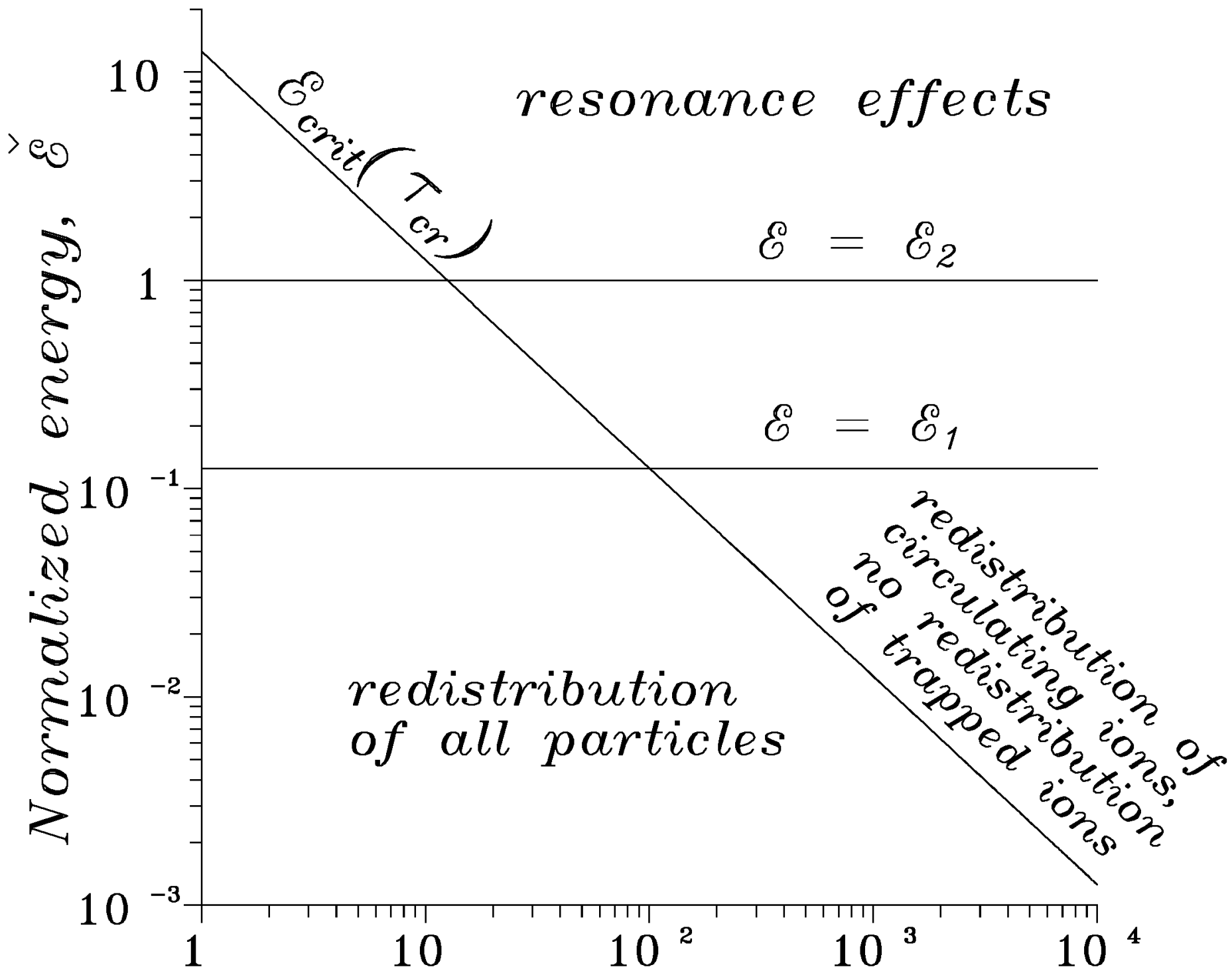

\section{Normalized crash duration, $\check{\tau}_{\text {cr }}$}

FIG. 8. Effects of sawteeth on different groups of ions depending on the normalized ion energy $\breve{\mathcal{E}}$ and the normalized crash duration $\check{\tau}_{c r}$. Notations: $\breve{\mathcal{E}}=\rho^{2} /\left(\epsilon_{s} r_{s}^{2}\right)$, $\check{\tau}_{c r}=\epsilon_{s}^{2} \omega_{B} \tau_{c r}, \rho$ is the Larmor radius of a fast ion, subscript ' $s$ ' labels values at the $q=1$ surface radius. 